\title{
Development of novel immunotherapy based on nanoparticle co-delivering PLK1 and PD-L1 inhibitors for lung cancer treatment
}

\author{
Moataz Reda \\ PDX Pharmaceuticals
}

Worapol Ngamcherdtrakul

PDX Pharmaceuticals

\section{Molly Nelson \\ PDX Pharmaceuticals}

\section{Natnaree Siriwon}

Rajamangala University of Technology Thanyaburi

\section{Ruijie Wang}

PDX Pharmaceuticals

\section{Husam Zaidan}

PDX Pharmaceuticals

\section{Daniel Bejan}

PDX Pharmaceuticals

\section{Sherif Reda}

PDX Pharmaceuticals

Ngoc Hoang

Oregon Health and Science University

Noah Crumrine

PDX Pharmaceuticals

Justin Rehwaldt

PDX Pharmaceuticals

\section{Akash Bindal}

Oregon Health and Science University https://orcid.org/0000-0002-9834-6979

\section{Gordon Mills}

Oregon Health and Science University https://orcid.org/0000-0002-0144-9614 Joe Gray

Oregon Health \& Science University https://orcid.org/0000-0001-9225-6756

Wassana Yantasee ( $\nabla$ yantasee@ohsu.edu )

Oregon Health and Science University 


\section{Article}

\section{Keywords:}

Posted Date: March 11th, 2022

DOI: https://doi.org/10.21203/rs.3.rs-142908/v2

License: (c) (i) This work is licensed under a Creative Commons Attribution 4.0 International License. Read Full License

Version of Record: A version of this preprint was published at Nature Communications on July 23rd, 2022. See the published version at https://doi.org/10.1038/s41467-022-31926-9. 


\section{Abstract}

Immune checkpoint inhibitors (ICls) targeting PD-L1 and PD-1 have improved survival in a subset of patients with advanced non-small cell lung cancer (NSCLC). However, only a minority of NSCLC patients respond to ICls, highlighting the need for superior immunotherapy. Herein, we developed a nanoparticlebased immunotherapy termed ARAC (Antigen Release Agent and Checkpoint Inhibitor) to enhance the efficacy of PD-L1 inhibitor. ARAC is a nanoparticle co-delivering PLK1 inhibitor (volasertib) and PD-L1 antibody. PLK1 is a key mitotic kinase that is overexpressed in various cancers including NSCLC and drives cancer growth. Inhibition of PLK1 selectively kills cancer cells and upregulates PD-L1 expression in surviving cancer cells thereby providing opportunity for ARAC targeted delivery in a positive feedback manner. ARAC reduced effective doses of volasertib and PD-L1 antibody by 5 -fold in a metastatic lung tumor model (LLC-JSP) and the effect was mainly mediated by CD8 + T cells. Notably, ARAC also showed efficacy in another lung tumor model (KLN-205), which does not respond to CTLA-4 and PD-1 inhibitor combination. Further, the nanoparticle construct was well-tolerated in non-human primates. This study highlights a rationale combination strategy to augment existing therapies by utilizing our nanoparticle platform that can load multiple cargo types at once.

\section{Introduction}

The emergence of immune checkpoint inhibitors (ICls) specifically antibodies targeting the PD-1/PD-L1 axis has reshaped the treatment landscape for various cancers including non-small cell lung cancer (NSCLC). PD-L1 expression on tumor cells inhibits tumor-directed cytotoxic CD8 + T cell activity by binding to PD-1 receptors on T cells and suppressing their function. ${ }^{1-3}$ Thus, PD-1/PD-L1 overexpression is a hallmark of the immunosuppressive environment displayed in several types of cancer. PD-1/PD-L1 blockade induces potent responses in patients; however, only $15-20 \%$ of patients respond, and many initial responders often relapse suggesting resistance mechanisms. ${ }^{4-6}$ PD-L1 expression has emerged as a biomarker to predict response to ICls as high levels of PD-L1 are often associated with more responsive treatment outcomes, ${ }^{7}$ while low levels of tumor infiltrating lymphocytes (TILs) are associated with lack of response or eventual resistance. ${ }^{7,8}$ Thus, combinatorial strategies employing PD-L1/PD-1 inhibitors with other therapeutics that 1) upregulate PD-L1 expression and/or 2) increase density of TILs have great potential to enhance the response rate of treatments, and identify more curative approaches. Another limitation is that systemic distribution of ICls can result in pathologic autoimmunity, leading to immunerelated adverse events (irAEs) that damage normal tissues. ${ }^{9}$ Therefore, strategies to localize treatment effects to the tumor and thus improve safety have important clinical relevance.

Polo-like kinase 1 (PLK1) is a critical mitotic kinase that is overexpressed in various cancers and provokes oncogenic properties. ${ }^{10}$ Previous studies have illustrated the potential of PLK1 inhibition as a strong therapeutic strategy and several PLK1 small molecule inhibitors have reached clinical trials. ${ }^{11}$ However, clinical utility of PLK1 inhibitors has yet to be realized due to dose-limiting toxicities and poor efficacy as a monotherapy; thus, alternative therapeutic strategies are needed to elicit the full potential of 
inhibiting PLK1. In addition to oncogenic functions, PLK1 also has roles in immune regulation in several types of cancer. ${ }^{12}$ In NSCLC, PLK1 expression is negatively correlated with immune scores, major histocompatibility complex (MHC) class I activity, and expression of TILs. ${ }^{12}$ PLK1 expression has been shown to be an activator of STAT3, which promotes an immunosuppressive environment. ${ }^{13}$ Inhibition of PLK1 results in reduced phosphorylation of STAT3, ${ }^{14,15}$ thereby dampening its activity in NSCLC cells. Furthermore, PLK1 inhibition has been shown to increase MHC class I expression on multiple cell lines, ${ }^{12}$ suggesting a role in adaptive immunity. In short, PLK1 expression contributes to immunosuppressive tumor microenvironment (TME), inhibiting immune cell infiltration and antitumor immunity. Thus, in addition to direct toxicity on cancer cells, inhibiting PLK1 may enhance antitumor immunity and have synergy with immunotherapy. Herein, we investigate the relationship of PLK1 inhibition with immune checkpoint blockade. We report for the first time that PLK1 inhibition upregulates PD-L1 expression of cancer cells, thereby diminishing cytotoxic T cell function. Accordingly, the combination of PD-L1 blockade with PLK1 inhibition significantly reduced tumor burden and prolonged survival of lung tumorbearing mice.

To facilitate clinical translation of this combination, we developed a nano-immunotherapy termed ARAC (Antigen Release Agent and Checkpoint inhibitor) for co-delivery of a PLK1 inhibitor (volasertib) and PDL1 antibody. ARAC is built upon our polymer-coated mesoporous silica nanoparticle (NP) platform, which has shown substantial promise as a tumor-targeted delivery vehicle. ${ }^{14,16-18}$ Indeed, we show that delivery of the therapeutic compounds (PLK1 inhibitor (volasertib) and PD-L1 antibody) with our NP platform can reduce the effective drug doses by 5 -fold in an aggressive metastatic lung tumor model. This is significant as it alleviates toxicity concerns of each drug, while achieving the therapeutic synergy of the drug combination. PLK1 inhibition induces cancer cell death while also upregulating PD-L1 expression of surviving cancer cells, thereby providing the opportunity to target surviving cancer with PD-L1 antibodyconjugated nanoparticles (benefiting cancer otherwise having no established receptors for drug delivery). The upregulation of PD-L1 expression also renders cancer more responsive to PD-1/PD-L1 blockade with PD-L1 antibody on ARAC. Cellular uptake of PD-L1 bound ARAC by endocytosis also lowers the level of membrane PD-L1 expression, allowing the cancer cells to be attacked by cytotoxic T cells. Our study provides evidence for the relationship between PLK1 inhibition and cancer immunosuppression and supports the combination of PLK1 inhibitor and PD-L1 immune checkpoint blockade as a potential therapeutic option. Further, our study highlights a rationale combination strategy to augment existing therapies without increasing toxicity by utilizing a nanoparticle platform as a delivery carrier.

\section{Results}

\section{PLK1 inhibition upregulates PD-L1 expression}

We and others have previously reported that PLK1 inhibition or knock-down results in a cell cycle arrest in G2/M phase leading to cancer cell death. ${ }^{14,19-22}$ Herein, we found that PLK1 knockdown also increases PD-L1 surface expression in both human (A549) and murine (LLC-JSP) lung cancer cell lines. As shown 
in Fig. 1A, 85\% knockdown of PLK1 mRNA (by siRNA against PLK1) resulted in 2.5-fold increase in PD-L1 mRNA expression in A549 cell line compared with untreated cells at 2 days after siRNA treatment. This was then confirmed at the surface protein level in A549 (Fig. 1B) and LLC-JSP (Fig. 1C) lung cancer cell lines at 3 days after siRNA treatments.

\section{Mitotic kinase inhibitor (MKI)-induced PD-L1 expression}

Following on the discovery that PLK1 inhibition results in PD-L1 upregulation, we sought to determine whether this holds true for inhibition of other mitotic kinases. We screened three mitotic kinase small molecule inhibitors against PLK1 (volasertib), Aurora kinase A (alisertib), and CHK1 (AZD7762) in human and mouse lung cancer cell lines. As shown in Supplementary Fig. 1, treatment of human NSCLC cells (A549 and H460) and a murine lung cancer cell (LLC-JSP) with volasertib, alisertib, or AZD7762 all reduced cancer cell viability and upregulated surface PD-L1 levels. These results establish the link between mitotic kinase inhibition and PD-L1 upregulation, and also demonstrate the potency of volasertib over other mitotic kinase inhibitors. In agreement with our results using PLK1 siRNA (Fig. 1) or volasertib (Supplementary Fig. 1), onvansertib (a newer PLK1 small molecule inhibitor) also reduced cell viability and upregulated PD-L1 expression in A549 cells (Supplementary Fig. 2).

\section{PLK1 inhibition leads to PD-L1 upregulation through activation of the mitogen-activated protein kinase (MAPK) pathway}

Previous studies have shown the involvement of the MAPK pathway and the NF-kB transcription factor in PD-L1 upregulation after paclitaxel (mitotic inhibitor chemotherapy) treatment. ${ }^{23,24}$ Specifically, Gong et al. reported that paclitaxel-induced PD-L1 expression was MAPK-dependent in colorectal and liver cancers, ${ }^{23}$ while Peng et al. found that paclitaxel induces PD-L1 upregulation via NF-kB in ovarian cancer. $^{24}$ To decipher the mechanism of PLK1 inhibition-induced PD-L1 expression, we probed downstream signaling pathways after PLK1 inhibition with volasertib. We found that volasertib led to activation of MAPK pathway, specifically with increase in phosphorylated ERK1/2 expression, and volasertib also led to an increase in phosphorylated NF-kB expression (Supplementary Fig. 3). In NSCLC, Jong et al. reported that the MAPK pathway plays a key role in PD-L1 regulation through increasing transcriptional activity and stabilizing PD-L1 (CD274) mRNA. ${ }^{25}$ Thus, we hypothesized that volasertib induced PD-L1 expression through the MAPK pathway, which is a known regulator of PD-L1 expression. ${ }^{26}$ To investigate this, NSCLC cells (H460) were treated with volasertib, ERK1/2 small molecule inhibitor SCH772984, or both drugs, and PD-L1 expression was assessed by flow cytometry. As shown in Fig. 2A, inhibition of ERK1/2 could negate the elevated PD-L1 expression induced by volasertib, as cells treated with both volasertib and SCH772984 showed similar PD-L1 expression level to vehicle control-treated cells. Moreover, since PLK1 inhibition also led to increased expression of phosphorylated NF-kB (a known transcription factor for PD-L1), NSCLC cells were treated with NF-kB inhibitor SC75741 in combination with volasertib, and PD-L1 expression was again assessed with flow cytometry. As shown in Fig. 2B, 


\section{Combination of PLK1 inhibition and PD-L1 blockade reduces tumor growth and prolongs survival in mice}

Based on our finding that PLK1 inhibition upregulated PD-L1 protein, we investigate whether the PLK1 inhibitor volasertib and a PD-L1 monoclonal antibody synergize in vivo. We used a LLC-JSP cell line to develop a flank tumor model in immune-competent mice similar to a previous report. ${ }^{27}$ Mice with tumors (>60 $\mathrm{mm}^{3}$ ) were treated i.p. with volasertib, PD-L1 antibody, or the combination of volasertib and PD-L1 antibody as shown in Fig. 3A. The combination treatment (volasertib + PD-L1 antibody) significantly reduced tumor growth (Fig. 3B) and prolonged survival of mice (Fig. 3C) better than each monotherapy, which did not provide a statistically significant survival effect compared with the vehicle-treated mice.

\section{PD-L1 antibody conjugated nanoparticles for delivery of PLK1 inhibitor volasertib (ARAC)}

Our data show for the first time the benefit of combining PD-L1 antibody and PLK1 inhibitor to enhance therapeutic impact. However, each drug alone carries significant toxicity risks that may limit the clinical translation of this combination. To overcome this limitation, we investigated a targeted delivery approach utilizing our polymer modified mesoporous silica nanoparticle (NP) platform. The same nanoparticle platform has been proven effective for targeted delivery of siRNA to breast tumors and lung tumors in our prior work. ${ }^{14,16,17,28}$ Volasertib (iPLK1) was loaded onto mesoporous silica nanoparticle (MSNP) core prior to surface modification with polyethylenimine (PEI), polyethylene glycol (PEG), and PD-L1 antibody (Fig. 4A). The final composition (p-iPLK1-NP or ARAC) contained 14.3\% PEI and 10\% PEG (by thermogravimetric analysis (TGA); Supplementary Fig. 4C), with $0.5 \%$ volasertib (by UV-Vis absorbance at $330 \mathrm{~nm}$ ), and 4.0\% PD-L1 antibody (by BCA assay) (by weight of MSNP). Volasertib loaded nanoparticles, with PD-L1 antibody (p-iPLK1-NP; ARAC) or without (iPLK1-NP), had a hydrodynamic size of $90 \mathrm{~nm}$ (Fig. 4B), and rigid core size of $50 \mathrm{~nm}$ by TEM (Supplementary Fig. 4A). In agreement with our prior studies, we found that the nanoconstruct can be stored stably at $-80{ }^{\circ} \mathrm{C}$ and retain efficacy and hydrodynamic size as fresh material (Supplementary Figs. 4B). Regarding drug release, we found that volasertib was preferentially released from the nanoconstruct in endo/lysosomal solution ( $\mathrm{pH} 4.5)$ over PBS (pH 7.4) (Supplementary Fig. 4D). As shown in Supplementary Fig. 5, ARAC significantly reduced cell viability of human (A549 and H460) and mouse (LLC-JSP) lung cancer cells in a dose-dependent manner, while the bare nanoparticle (NP) showed minimal toxicity. Moreover, treatment of LLC-JSP cells with iPLK1-NP significantly reduced cell viability more than the free volasertib counterpart treated at the same dose (Fig. 4C). Similar results were obtained with B16-F10 melanoma cells and 4T1 breast cancer cells (Supplementary Fig. 6) suggesting broad efficacy of the material. In vitro, PD-L1 antibody (see ARAC vs. iPLK1-NP; Fig. 4D) had no role in cancer killing (since there were no T cells in the system) or enhancing the delivery (since all nanoparticles were taken up by cells within 3 days regardless of having PD-L1 
antibody or not). In agreement with previous finding using PLK1 siRNA (Fig. 1) or free inhibitors, treatment with iPLK1-NP resulted in significant increase in surface PD-L1 expression of the surviving cells at 2 days (about $60 \%$ cells were dead), but not at 2 hrs (Fig. 4E) since PLK1 inhibition effects (i.e. cell cycle arrest and death) had not yet transpired. However, PD-L1 antibody on the nanoparticles was as effective as free PD-L1 antibody, given at a 30-fold higher dose, at reducing surface staining of PD-L1 level (see ARAC at 2 hrs, Fig. 4E), which may be attributed to blockade by the therapeutic antibody or PDL1 internalization along with the nanoparticles (Supplementary Fig. 7). This is owing to the high local concentration of antibodies on the nanoparticles that the cells encountered. Nanoparticles with dense PDL1 antibodies (approximately $2 \times 10^{3}$ antibodies per particle) can bind multiple PD-L1 ligands on cell surface at once and endocytose with PD-L1 (termed receptor-meditated endocytosis). ${ }^{29}$ Furthermore, Fig. 4F suggests that PD-L1 upregulation in the surviving population upon treatment with iPLK1-NP is not via selection process (e.g., death of cells with low PD-L1 first) since untreated cells (PBS) did not have any high PD-L1 population), but rather is due to signaling effects of PLK1 inhibition. Indeed, PLK1 inhibition also led to upregulation of PD-L1 expression in melanoma and breast cancer cells with varying PD-L1 baseline expression (Supplementary Fig. 6).

\section{Uptake, feed-forward delivery, and specificity of ARAC nanoconstruct}

To study the uptake of the nanoconstruct, NSCLC cells were treated with PD-L1 antibody-conjugated nanoparticles (p-NP) carrying a fluorescent dye (Dy677) and incubated at $4^{\circ} \mathrm{C}$ for $1 \mathrm{hr}$ or $37^{\circ} \mathrm{C}$ for $1-4 \mathrm{hr}$ prior to imaging. As shown in Supplementary Fig. 7, most p-NP was effectively internalized into NSCLC cells ( $\mathrm{H} 460)$ upon $1 \mathrm{hr}$ incubation at $37^{\circ} \mathrm{C}$, while most $\mathrm{p}-\mathrm{NP}$ stayed on the cell membrane without any significant cellular uptake upon $1 \mathrm{~h}$ incubation at $4^{\circ} \mathrm{C}$. This suggests that active endocytosis, as opposed to passive uptake, ${ }^{30}$ is the primary uptake mechanism of p-NP. Further, higher PD-L1 staining is observed intracellularly upon $1 \mathrm{hr}$ incubation at $37^{\circ} \mathrm{C}$ compared with $1 \mathrm{hr}$ incubation at $4^{\circ} \mathrm{C}$, suggesting that PD-L1 is internalized with the nanoparticles. Moreover, since most p-NPs are intracellular at $1 \mathrm{hr}$, the observed lower surface PD-L1 expression reported in Fig. 4E (at $2 \mathrm{hr}$ time-point) is primarily due to PD-L1 internalization and not due to the nanoparticles' blocking the PD-L1 staining antibody on the cell membrane.

While ARAC initially engages PD-L1 upon binding and internalization (as shown in Fig. 4E and Supplementary Fig. 7), surviving cells have upregulated PD-L1 due to the signaling effects of PLK1 inhibition. In this context, upregulated PD-L1 is used as the homing target for subsequent ARAC, leading to cancer targeting in a feedforward manner (i.e., higher targeting with increased doses of the treatment). To investigate the feedforward targeting of ARAC, we used 4T1 murine cancer cells which express low baseline PD-L1 levels (lowest of any cancer cell line tested in our study). ARAC led to the upregulation of PD-L1 in 4T1 cells 4 days post treatment (Supplementary Fig. 8A). We then assessed the cellular uptake of ARAC in control 4T1 cells (with low PD-L1) and ARAC-treated 4T1 cells (with upregulated PD-L1). As shown in Supplementary Fig. 8B, after 1 hour exposure, ARAC was preferentially taken up by the PD-L1 
high cells vs. PD-L1 low cells by nearly 4 -fold, demonstrating the selectivity and feed-forward targeting by ARAC. We also evaluated the cell killing selectivity by comparing viability of murine cancer cells (LLC-JSP, 4T1, B16-F10) vs. bone marrow-derived dendritic cells (BMDC) after treatment with ARAC. As shown in Supplementary Fig. 8C, ARAC led to significant cell killing in cancer cells but minimal killing in dendritic cells. Similar to Fig. 4D, PD-L1 antibody has no effect on enhancing the delivery in this setting since all nanoparticles are taken up by cells within 3 days regardless of PD-L1 expression. Thus, the treatment selectivity to cancer cells over BMDC cells is due to cancer dependence on PLK1, as previously reported. ${ }^{31}$

\section{ARAC induces anti-tumor immune response in a bilateral lung cancer tumor model}

To assess the anti-tumor immune effect of ARAC, we utilized a bilateral flank tumor model. C57BL/6 mice were injected with 100,000 and 40,000 LLC-JSP cells on the right and left flank, respectively. At day 12 post injection, mice were grouped $(n=7)$ and the right flank (local) tumors were injected with PBS, $p-N P$ (NP with PD-L1 antibody), iPLK1-NP (NP with volasertib), or ARAC as shown in Fig.5A.Growth of local (treated) and distant (untreated) tumors were monitored. Treatments with ARAC significantly reduced growth of local tumors compared with p-NP or iPLK1-NP (Fig.5B). We did not observe significant tumor reduction with iPLK1-NP since the volasertib administered in this study $(0.125 \mathrm{mg} / \mathrm{kg}$ intratumoral for 3 doses) was much lower than the efficacious dose range reported for volasertib in xenograft tumors (e.g. $10-40 \mathrm{mg} / \mathrm{kg}$ once or twice a week systemically). ${ }^{32-34}$ Similarly, the PD-L1 antibody dose on p-NP (20 $\mu \mathrm{g}$ for 3 doses) is lower than the efficacious dose (200 $\mu \mathrm{g}$ i.p. once a week) reported in this model. As shown in Fig.5C, a delay in the onset of distal tumors was also observed in ARAC group, suggesting that a systemic anti-tumor immune response was generated. Further, ARAC significantly prolonged survival of mice vs. saline or single drug NPs (Fig.5D). In a separate study, mice were inoculated with 250,000 and 100,000 LLC-JSP cells for bilateral tumors and treated as shown in Fig.5A with ARAC or saline. Tumors and tumor-draining lymph nodes were harvested one day post last treatment for immune profiling with flow cytometry. As shown in Fig.5E, PD-L1 surface expression was reduced in ARAC-treated tumors in both hematopoietic (CD45+, primarily immune cells) and nonhematopoietic (CD45-, primarily cancer cells) cell populations, confirming the efficacy of PD-L1 antibody on the nanoconstruct. ARAC also significantly enhanced proliferation (Ki67+) of effector CD8+T cells in the local tumor-draining lymph nodes (Fig.5F). Longer time-point (than one day post dosing) may be needed to see significant changes in distant tumors to decipher the observed delayed onset of the distant tumors in Fig.5C. Importantly, ARAC-treated tumors had significantly higher population of total immune cells (CD45+) and CD8+T cells, and significantly higher ratio of CD8+/Treg ratio as compared with control tumors (Fig.5G).

To evaluate ARAC systemically, we developed an experimental metastatic lung tumor model by intravenous injection of LLC-JSP cells $(200,000$ cells), which developed tumors mainly in the lungs (confirmed at sacrifice). Mice were randomly grouped and treated intravenously (i.v.) via tail vein with saline, free drugs (volasertib + PD-L1 antibody at same dose or 5-fold higher dose than dose on ARAC), ARAC, or ARAC plus anti-CD8 antibody (Fig. 6A). Mice treated with ARAC survived significantly longer than those treated with saline or free drugs at same dose (*** $p<0.001$ vs. saline; ${ }^{* \star} p<0.01$ vs. free drugs 
(1x)) (Fig. 6B) and slightly better than those treated with the 5 -fold dose of the free drug combo $\left({ }^{*} p<0.05\right.$ vs. saline). Thus, delivery with ARAC could effectively reduce required dose of drug by at least 5-fold. Moreover, ARAC's efficacy was confirmed to be immune-mediated as CD8 + T cell depletion by anti-CD8 antibodies abolished the prolonged survival of ARAC-treated mice (Fig. 6C). Furthermore, treatment with ARAC did not cause any weight loss, demonstrating its favorable safety in mice (Fig. 6D).

\section{ARAC efficacy in ICI-refractory KLN-205 syngeneic tumor model}

We further evaluated ARAC's in vivo efficacy in an ICl-refractory tumor model, $\mathrm{KLN}-205 .{ }^{35,36}$ We compared ARAC efficacy to dual ICI treatments of PD-1 and CTLA-4 antibodies (which was recently granted FDA approval for metastatic NSCLC). DBA/2 mice were inoculated with 500,000 KLN-205 cells on the right flank. When tumors developed $\left(\sim 40 \mathrm{~mm}^{3}\right)$, mice were grouped $(\mathrm{n}=7)$ and treated i.v. with $50 \mathrm{mg} / \mathrm{kg}$ ARAC (for 4 doses on days 0, 3, 9, 12), i.p. with ICls (PD-1 and CTLA-4 antibodies - $200 \mu \mathrm{g} /$ dose and 100 $\mu \mathrm{g} /$ dose respectively - for 6 doses on days $0,3,9,12,21,28)$, or saline. As shown in Fig. 7A, ARAC treatments significantly reduced tumor growth vs. saline ( $\left.{ }^{\star \star p} p<0.01\right)$ and vs. ICls $\left({ }^{\star *} p<0.01\right)$, while ICls had no effect on tumor growth vs. saline. Moreover, mice treated with ARAC survived significantly longer than saline or ICl-treated mice (Fig. 7B).

\section{Safety of nanoparticle platform in cynomolgus monkeys}

To date, we found our NP platform to meet required safety criteria: (1) Low cytotoxicity of multiple organ cells (< $10 \%$ cell death), ${ }^{28}$ (2) Great blood compatibility, ${ }^{16}$ (3) Not triggering adverse immune response of blood immune cells (PBMC), ${ }^{16}$ (4) Excellent safety after 7 doses given systemically to mice over 1 month by not causing adverse effects to body weight, serum biomarkers, and histology of kidney and liver, ${ }^{18}(5)$ Good maximum tolerated dose (MTD not reached at 4 -fold of efficacious dose), ${ }^{18}$ and (6) effective clearance as MSNP is soluble to benign silicic acid ${ }^{37,38}$ at serum $\mathrm{pH}$ and cleared in urine. ${ }^{39,40}$ Herein, we report preliminary toxicology study of the NP platform co-delivering a PLK1 siRNA (as an alternative for volasertib) and PD-L1 antibody (avelumab), namely p-siPLK1-NP, in non-human primates (NHP). We aim to replace volasertib with PLK1 siRNA as we progress towards clinics since the NP construct has shown outstanding efficacy in delivery siPLK1 to tumors in lungs ${ }^{14,17}$ and we previously found that cancer was less prone to develop resistance to siRNA than to small molecule inhibitor counterparts. ${ }^{41}$ As shown in Supplementary Fig. 9, we also confirmed that PD-L1 antibody-conjugated NP could effectively deliver PLK1 siRNA and reduce cell viability of NSCLC cells. To study the toxicity of this nanoconstruct, cynomolgus monkeys $(n=3)$ received intravenous infusion of $5.6 \mathrm{mg} / \mathrm{kg}$ bare NP, $6 \mathrm{mg} / \mathrm{kg} \mathrm{p}$-siPLK1-NP (estimated efficacious dose ${ }^{42}$ ) and $18 \mathrm{mg} / \mathrm{kg} \mathrm{p}$-siPLK1-NP (3-fold efficacious dose), with a one week washout period in between dosing. Clinical signs, body weights, food consumption, dermal observations, clinical pathology parameters (hematology, coagulation, clinical chemistry, and cytokine secretion), gross necropsy findings, organ weights, and histopathologic examinations were evaluated. There were no test article-related clinical observations or effects on body weight (Supplementary Table 1), food 
consumption, or coagulation (Supplementary Table 3). Dermal observations of erythema and edema were noted at 6 and 24 hours post dose but resolved at 48 hrs post dose (Supplementary Table 6). For hematology, nonadverse decreased white blood cell (WBC), neutrophil, and lymphocyte counts were observed in 2 of 3 monkeys at the highest dose (18 mg/kg p-siPLK1-NP) on day 2 post dose but were resolved by day 7 post dose, indicating recovery (Supplementary Table 2). For clinical chemistry, nonadverse increased aspartate aminotransferase (AST) and alanine aminotransferase (ALT) levels were noted on day 2 following administration of NP alone, $6 \mathrm{mg} / \mathrm{kg} \mathrm{p}$-siPLK1-NP, and $18 \mathrm{mg} / \mathrm{kg}$ p-siPLK1-NP. However, for each group, AST and ALT levels of day 7 were similar to predose values, indicating recovery (Supplementary Table 4). Further, key cytokines (IL-1 $\beta$, IL-6, IFN- $\gamma$, TNF-a, IFN-a, and MCP-1) were monitored in serum collected at $0,6,24$, and 48 hours. Even in monkeys receiving the highest dose (18 $\mathrm{mg} / \mathrm{kg}$ ), only MCP-1 and IFN-a were mildly to moderately elevated in 2 out of 3 monkeys at $6 \mathrm{hrs}$, which subsided at 24 hrs without intervention (Supplementary Table 5). Increase of these cytokines right after treatment may be part of therapeutic actions (e.g., IFN-a suggests induction of innate immunity by siRNA; $;{ }^{43,44}$ PD-L1 inhibition induced higher production of MCP-1). ${ }^{45}$ Terminal euthanasia and necropsy was performed one week following the $18 \mathrm{mg} / \mathrm{kg}$ dosing to assess gross pathology, organ weights, and histopathology. A few observations were noted that were considered incidental and of the nature commonly observed in this species and age of monkeys. Thus, there were no test article-related effects on survival, organ weights, gross pathology, or histopathology (Supplementary Tables 7-9). In conclusion, the nanoconstruct was found to be safe and well tolerated in NHP at up to 3-fold anticipated efficacious dose.

\section{Discussion}

The potential of PLK1 inhibition as a therapeutic strategy has been well studied in various cancer types; however, the interplay of PLK1 inhibition with cancer immunity remains mostly unexplored. In this study, we report for the first time that PLK1 inhibition results in an increase of immune checkpoint PD-L1 expression in cancer cells. In NSCLC, PLK1 inhibition-induced PD-L1 upregulation was driven by the MAPK pathway, as inhibition of ERK1/2 could abolish the observed PD-L1 upregulation by volasertib. The upregulation of PD-L1 suggests that evading the immune response is one of the mechanisms exploited by cancer cells that survive PLK1 inhibition. Consequently, we show that the combination of PLK1 inhibitor and PD-L1 antibody significantly reduced tumor progression in mice compared to each drug alone. To the best of our knowledge, this is the first study to report the effectiveness of PLK1 inhibition in combination with any immunotherapy.

To overcome the dose limiting toxicity of current PLK1 inhibitors that prevent them from advancing beyond clinical trials, we developed a PLK1 inhibitor loaded nanoparticle platform and conjugated it to PD-L1 antibody (ARAC) to synergize combination effects of PLK1 inhibition and PD-L1 blockade. In our prior works, we reported on the efficacy and safety profile of this MSNP platform in delivering siRNA to mediate gene knockdown of breast and lung tumors in vivo. ${ }^{14,17}$ The sol gel MSNP synthesis and the layer-by-layer modification on MSNPs offer good synthesis reproducibility and scalability. We have 
successfully scaled up the synthesis protocol to yield $16 \mathrm{~g}$ of nanoparticles (2.5-L reaction volume), which is over 100 -fold higher than our small-scale synthesis. ${ }^{46}$ We have also reported excellent batch-tobatch reproducibility of the nanoconstruct synthesis for chemical and physical properties and bioactivities. ${ }^{14,16}$ Regarding stability, we found that the nanoparticles are stable at $-20^{\circ} \mathrm{C}$ for at least 4 months (longer time is being investigated) and $-80^{\circ} \mathrm{C}$ for years in terms of size and efficacy. ${ }^{39}$ We have also previously reported that our antibody-conjugated nanoparticles can be lyophilized, which maintains characteristics and efficacy over time. ${ }^{46}$

In this research, we demonstrate that the nanoparticle platform can also improve delivery of small molecule inhibitors (i.e. volasertib) as treatment with PLK1 inhibitor on nanoparticles significantly reduced cell viability compared with free PLK1 inhibitor. In mice bearing bilateral tumors, 3 doses of intratumoral ARAC reduced growth of local (injected) tumors compared with nanoparticle delivering a single drug, and significantly delayed the onset of distant tumors suggesting that a systemic anti-tumor response was triggered. Immune profiling of tumors showed that ARAC could effectively reduce PD-L1 expression in both immune (CD45+) and cancer cells (CD45-). Further, ARAC resulted in significant increase in CD8 + tumor infiltrating lymphocytes, as well as increase in CD8+/Tregs ratio, indicating that the treatment could reshape the tumor microenvironment to an immune-permissive state. Further, the therapeutic benefit of nanoparticle delivery was demonstrated in an experimental metastatic lung tumor model, where i.v. administration of ARAC improved survival as much as the free drugs at 5-fold higher dose. This suggests that nanoparticle delivery can overcome dose limiting toxicity issues of PLK1 inhibitors and thereby facilitate clinical utility. ARAC's efficacy was confirmed to be immune-mediated as CD8 depletion abolished the prolonged survival. Importantly, in an ICl-refractory tumor model, ARAC retained effectiveness and significantly reduced tumor growth and prolonged survival of mice, which suggests that ARAC may overcome the clinical limitation of immune checkpoint inhibitors. Furthermore, i.v. administration of the nanoparticle construct was found to be well tolerated and safe to cynomolgus monkeys at up to 3-fold estimated efficacious dose in a preliminary toxicology study.

Mechanistically, ARAC leads to cell cycle arrest and generation of an anti-tumor immune response, while exhibiting unique feed-forward delivery capability (e.g., greater delivery to surviving cancer cells having upregulated PD-L1 levels from an initial treatment) to mount an anti-tumor immune attack (Scheme 1). For tumors with initially low PD-L1 levels, ARAC with slight positive charge ( $6 \mathrm{mV}$ in $10 \mathrm{mM} \mathrm{NaCl})$ may rely first on enhanced permeability and retention effect (EPR) and adsorptive endocytosis and subsequently on PD-L1 antibody mediated endocytosis after volasertib-induced PD-L1 upregulation in the surviving cancer cells. While toxic to multiple cancer cells, ARAC is safe to antigen-presenting cells (DCs), needed for priming anti-tumor T cells.

Our research herein focused on lung cancer, the leading cancer killer. ${ }^{47}$ Like melanoma, where immunotherapy has been the most promising, lung cancer is a disease with a high mutational load, which drives the expression of various neo-epitopes that can be recognized by host immune system. ${ }^{48,49}$ Consequently, immunotherapy is a promising approach to treat lung cancer. However, objective response 
rates are much lower for lung cancer patients than melanoma. The research described here illustrates how superior responses can be achieved for lung cancers when combining PLK1 inhibition with PD-L1 blockade. Further, other cytotoxic agents have also been shown to increase PD-L1 expression, including paclitaxel in ovarian cancer, ${ }^{24}$ CDK4/6 inhibitors, ${ }^{50}$ and PARP inhibitors ${ }^{51}$ in breast cancer. Therefore, it is logical that these drugs are now in clinical investigations in combination with PD-1/PD-L1 checkpoint blockade. ${ }^{52}$ Our findings suggest that co-delivering these drugs with PD-L1 immune checkpoint antibody on our nanoparticle construct will increase efficacy while lowering toxicity. Further, as PLK1 overexpression has been found in a variety of cancers, the combination of PLK1 inhibition and PD-L1 blockade may have broad application to a plethora of cancers. Lastly, other mitotic kinase inhibitors that elevate PD-L1 expression of cancer cells should also be combined with PD-L1 immune checkpoint blockade to improve treatment outcomes in clinics.

\section{Methods}

\section{Cell lines and reagents}

A549 and H460 NSCLC cells were purchased from ATCC and maintained in RPMI media with $10 \%$ fetal bovine serum (FBS). Mouse cancer cell lines KLN205, B16F10, and 4T1 were purchased from ATCC and maintained in EMEM + 10\% FBS, DMEM + 10\% FBS, and RPMI + 10\% FBS, respectively. Lewis Lung Carcinoma (LLC) metastatic variant, LLC-JSP cells were gift from Dr. Don Gibbons lab (MD Anderson Cancer Center), and were cultured in RPMI $+10 \%$ FBS. BMDCs were harvested from naïve mice and cultured following published protocols. ${ }^{53,54}$ Staining antibodies used: human PD-L1 (PE; clone MIH1, Biolegend), mouse PD-L1 (PE; clone MIH5, BD Biosciences), mouse CD8 (BV650; clone 53 - 6.7, BD Biosciences), mouse CD4 (BV711; clone RM4-5, BD Biosciences), mouse CD45 (APC-Cy7; clone 30-F11, Biolegend), mouse CD3 (PerCP5.5; clone 17A2 Biolegend), mouse/human CD44 (FITC; clone IM-7 Biolegend), mouse Ki-67 (efluor450; clone SolA15, invitrogen), mouse FoxP3 (Alexa-647; clone MF-14, Biolegend), human PD-L1 antibody (unconjugated; clone MIH1, eBioscience). Alexa Fluor 488 secondary antibody was purchased from Life Technologies. In vivo grade (InVivoMab) anti-mouse PD-L1 antibody (BE0101; clone 10F.9G2), anti-mouse PD-1 antibody (BE0146; RMP1-14), anti-mouse CTLA-4 antibody (BE0131; clone 9H10), and anti-mouse CD8a antibody (BE0061; clone 2.43) were purchased from BioXcell. Pharmaceutical-grade human PD-L1 antibody avelumab (Pfizer/Merck KGaA) was purchased from OHSU Pharmacy. Small molecule inhibitors (volasertib, alisertib, AZD7762, onvansertib, SC75741, SCH772984) were purchased from Selleckchem. All inhibitors were solubilized in DMSO at high concentrations and diluted down to $1 \%$ DMSO (in PBS pH 7.2) for working concentrations - final DMSO content in wells (exposed to cells) was 0.1\% DMSO. SiRNA sequences: PLK1 (antisense 5'-

UAUUCAUUCUUCUUGAUCCGG-3'); scrambled SCR (antisense 5'-UUAGUCGACAUGUAAACCA-3') and Dharmafect 1 Transfection Reagent were purchased from Dharmacon (Horizon).

\section{Nanoparticle synthesis and characterization}


Bare MSNPs were synthesized as we have previously reported. ${ }^{16}$ For PLK1 inhibitor loading, volasertib was mixed with MSNPs in ethanol for overnight shaking at room temperature (350 RPM). The next day, nanoparticles were coated with $10 \mathrm{kDa}$ branched PEI (Alfa Aesar) and 5 kDa mal-PEG-NHS (Jenkem) following our previous studies. ${ }^{16,46}$ For PD-L1 antibody conjugation, in vivo grade mouse PD-L1 antibody (BioXcell) or avelumab was buffer exchanged to PBS pH 8 (Zeba spin column, Thermo Fisher) and thiolated using Pierce Traut's reagent (Thermo Fisher) following manufacturer's protocol. Thiolated antibody was added to NP at $20 \mathrm{wt} . \%$ and shaken overnight at $4^{\circ} \mathrm{C}$ (300 RPM). Nanoparticles were washed with PBS pH 7.2 before characterization. To determine polymer loading and final nanoparticle concentration, $1 \mathrm{mg}$ nanoparticles were heated to $900^{\circ} \mathrm{C}\left(20^{\circ} \mathrm{C} / \mathrm{min}\right)$ using TGA Q50 (TA Instruments). Nanoparticle size was determined using Zetasizer (Malvern, ZS-90). Antibody loading was 4 wt.\%, determined by protein quantification of NP supernatant with Pierce BCA protein assay (Thermo Fisher). To quantify PLK1 inhibitor loading, nanoparticles were shaken in DMSO solution (75\% DMSO, 25\% PBS) for $24 \mathrm{hr}$ to release the drug and supernatant was collected. Absorbance $(330 \mathrm{~nm})$ of supernatant was measured with Infinite 200 Pro plate reader (Tecan) to determine loading extent. siRNA was loaded onto the nanoparticles by 5-min mixing in PBS at room temperature, and complete loading was confirmed by Nanodrop Spectrophotometer (ND-1000, Thermo Fisher). For drug release study over time, nanoparticles were incubated in PBS pH 7.4 (1X, Gibco) or lysosomal simulant solution pH $4.5(100 \mathrm{mM} \mathrm{NaCl}, 100 \mathrm{mM}$ $\mathrm{KCl}, 100 \mathrm{mM}$ sodium acetate; acidified to $\mathrm{pH} 4.5$ with $1 \mathrm{M} \mathrm{HCl}$ ) at a final NP concentration of $4 \mathrm{mg} / \mathrm{mL}$. Samples were shaken on a temperature controlled orbital shaker at $100 \mathrm{RPM}, 37^{\circ} \mathrm{C}$. At indicated time points, aliquots were removed and centrifuged to pellet the nanoparticles and supernatant was collected to quantify volasertib released by UV-vis (absorbance at $330 \mathrm{~nm}$ ). For Transmission electron microscopy (TEM), 5uL of nanoparticle preparations were deposited onto glow discharged (60 secs 15 mAmp, negative mode) carbon formvar 400 Mesh copper grids (Ted Pella 01822-F) for 3 min, rinsed 15 secs in water, wicked on Whatman filter paper 1, stained for $3 \mathrm{~min}$ in filtered $1 \%(\mathrm{w} / \mathrm{v})$ uranyl acetate in water, wicked and air dried. Samples were imaged at $120 \mathrm{kV}$ on a FEI Tecnai ${ }^{\mathrm{TM}}$ Spirit TEM system. Images were acquired using the AMT software interface on a Nanosprint12S-B cMOS camera system.

ARAC refers to nanoparticle loaded with both PLK1 inhibitor (volasertib) and PD-L1 antibody, p-NP is nanoparticle loaded with PD-L1 antibody, and iPLK1-NP is nanoparticle loaded with PLK1 inhibitor. For the majority of studies (in mice or using murine cell lines), in vivo grade mouse PD-L1 antibody (clone 10F.9G2; BioXCell) was used. For all studies using human cell lines and in nonhuman primates, avelumab (Pfizer/Merck kGAa) was used as the PD-L1 antibody on NP.

\section{Flow cytometry}

For in vitro studies, cells were plated in 6-well plates and treated with indicated treatments. 3 days post treatments, cells were collected and washed in FACS buffer prior to staining. Primary (unconjugated or fluorophore-conjugated) and secondary antibodies were stained for 30 mins and 1 hour, respectively, under rocking on ice. After staining, cells were washed in FACS buffer before analysis with Guava easyCyte (Millipore Sigma) flow cytometer (10,000 events per sample). For immune profiling, tumors and tumor-draining lymph nodes were harvested and cut into small sections for digestion. Tissues were

Page $13 / 26$ 
digested in digestion media ( $1 \mathrm{mg} \mathrm{mL}-1$ Collagenase $\mathrm{D}$ and $0.1 \mathrm{mg} \mathrm{mL}-1 \mathrm{DNase}$ I in HBSS) at $37^{\circ} \mathrm{C}$ for $30 \mathrm{~min}$ and mechanically dissociated by passing through $70 \mu \mathrm{m}$ pore nylon cell strainers. Red blood cells in the sample were lysed by incubating in RBC lysis buffer (Alfa Aesar) at room temperature for 5 min. Cells were washed twice with PBS and stained with Live/Dead Fixable Aqua Stain (Thermo Fisher Scientific) for $15 \mathrm{~min}$. Cells were washed twice with FACS buffer (1\% BSA in PBS), incubated with FcR blocking solution for $5 \mathrm{~min}$, and then stained for a select panel of surface-staining antibodies for $15 \mathrm{~min}$ at room temperature. Intracellular staining (for FoxP3 and Ki67) was performed with BD Cytofix/Cytoperm (BD Biosciences), following the manufacturer's protocol after cell surface staining. Samples were washed twice with FACS buffer and resuspended in FACS buffer for analysis. All data were acquired with a BD LSRFortessa flow cytometer (OHSU's Flow Cytometry Core), and analyzed using FlowJo Software (TreeStar Inc.). Only live cells (determined by live-dead stain occurring before fixing/permeabilization) were analyzed.

\section{Cell viability after treatments}

Cells (1500/well) were plated in white flat bottom 96 well plate overnight. The following day, cells were treated with small molecule inhibitors or drug loaded nanoparticles and controls as indicated and media was changed $24 \mathrm{hr}$ post treatment. 3 days post treatment, cell viability was assessed using Cell Titer Glo assay (Promega) following manufacturer's instructions. Luminescence was read with Tecan plate reader.

\section{RT-qPCR to assess PLK1 and PD-L1 mRNA}

Cells were seeded in 6-well plates and treated with PBS, siSCR, or siPLK1 using Dharmafect 1 Transfection Agent (Horizon), and media was changed after $24 \mathrm{hr}$. Two days post transfection, RNA was isolated with GeneJet RNA purification kit (Thermo Fisher Scientific) following manufacturer's instructions. One-Step qRT-PCR was performed using EXPRESS One-Step Superscript ${ }^{\text {TM }}$ qRT-PCR Kit (Invitrogen). Cycling conditions: $50^{\circ} \mathrm{C}$ for $2 \mathrm{~min}, 95^{\circ} \mathrm{C}$ for $10 \mathrm{~min}, 40$ cycles of $95^{\circ} \mathrm{C}$ for $15 \mathrm{~s}$, and $60^{\circ} \mathrm{C}$ for 1 min. TAQMAN gene expression primers Human HPRT mRNA (Hs99999909_m1), Human PLK1 mRNA (Hs00983225_g1), and Human PDL1 (Hs00204257_m1) were used. Data was analyzed using $2^{-\triangle \Delta C(t)}$ method.

\section{Western blot}

NSCLC cells were seeded in $100 \mathrm{~mm}$ dishes (300,000 cells/dish) overnight and treated with indicated treatments. Cell culture medium was changed one day after treatment. Three days post treatment, cells were lysed in RIPA buffer. Lysate was sonicated and centrifuged (15,000 RPM for 15 minutes) and supernatant was collected. Amount of total protein was quantified using BCA. $30 \mu \mathrm{g}$ of proteins (per sample) were mixed with 4 X Novex NuPAGE LDS sample buffer and beta-mercaptoethanol ( $10 \%$ final concentration). Samples were denatured for $5 \mathrm{~min}$ at $95^{\circ} \mathrm{C}$ and loaded onto gel (NuPAGE) for electrophoresis. Proteins were then transferred onto PVDF-FL membrane and blocked with LI-COR Intercept (TBS) blocking buffer. Membranes were incubated with primary antibodies overnight (PhosphoNF-kB p65 (Ser536) (93H1) antibody \#3033, Phospo-p44/42 MAPK (ERK1/2) (Thr202/Tyr204) antibody $\# 9101, \beta$-Actin (8H10D10) antibody \#3700) at $4^{\circ} \mathrm{C}$. Next day, membranes were rinsed with TBS-T and 
IRDye conjugated secondary antibodies (LI-COR) were added for 1 hour under rocking at room temperature. Membranes were scanned on a LI-COR Odyssey imaging system.

\section{Syngeneic tumor models and treatments}

For single tumor LLC-JSP model (Fig. 2), LLC-JSP murine lung cancer cells $(200,000)$ were inoculated in right flank of C57BL/ 6 female mice ( 6 weeks) (Charles River $\mathrm{NCl}$ colony). At 8 days post tumor inoculation, mice received intraperitoneal (i.p.) treatments of volasertib $(20 \mathrm{mg} / \mathrm{kg})$ and/or PD-L1 antibody $(10 \mathrm{mg} / \mathrm{kg}$ ) every 5 days for 3 doses total. For bilateral LLC-JSP tumors, C57BL/ 6 were inoculated with 100,000 and 40,000 LLC-JSP cells in right and left flank, respectively. At 12 days post inoculation, the aforementioned treatments were administered intratumorally to the right tumor every 3 days for 3 doses total. For KLN205 tumors, DBA/2 mice (Charles River NCl colony) were inoculated in right flank with $500,000 \mathrm{KLN} 205$ cells. Starting at 13 days post inoculation, mice received treatments of saline (i.v.), ARAC (i.v.), or PD-1 and CTLA-4 antibodies (i.p.) on indicated days. Tumors were measured with Vernier Caliper and volume calculated by $V=0.5 x$ length $x$ width ${ }^{2}$. For both single flank and bilateral flank tumor models, mice were sacrificed when total tumor burden exceeded $2000 \mathrm{~mm}^{3}$. For metastatic lung tumor model, LLC-JSP $(200,000)$ were injected intravenously (i.v.) to 6 week old C57BL/ 6 mice. At 3 days post cancer cell injection, mice were randomly grouped and treated with i.v. saline, i.v. ARAC (25 $\mathrm{mg} / \mathrm{kg} \mathrm{NP}$ ), i.v. ARAC plus i.p. CD8 antibody (10 mg/ $\mathrm{kg}$; twice a week), or i.p. PD-L1 antibody ( $5 \mathrm{mg} / \mathrm{kg}$ or $1 \mathrm{mg} / \mathrm{kg})$ plus volasertib $(0.625 \mathrm{mg} / \mathrm{kg}$ or $0.125 \mathrm{mg} / \mathrm{kg})$ every 3 days for a total of 4 doses. All studies were reviewed and approved by Institutional Animal Care and Use Committee (IACUC) at Oregon Health and Science University (OHSU).

\section{Statistical analysis}

GraphPad Prism 8.0 (GraphPad Software Inc.) was used for all statistical analysis. Comparison between two groups was performed with Student's $t$ test. Comparison between more than two groups was performed with One-Way ANOVA with Tukey's correction for multiple comparisons. Tumor growth was analyzed using two-way repeated measures ANOVA with Tukey's correction for multiple comparisons. Kaplan Meier survival curve was analyzed using the log-rank (Mantel-Cox) method. Significance was set at $\mathrm{p}<0.05$. In vitro data are expressed as mean $\pm \mathrm{SD}$; in vivo data are expressed as mean $\pm \mathrm{SEM}$.

\section{Declarations}

\section{Acknowledgement}

This work was funded by the Wayne D. Kuni \& Joan E. Kuni Foundation, National Cancer Institute of the National Institutes of Health (NIH) under Award Number R44CA217534, R44CA265751, and R44CA265752, National Center for Advancing Translational Sciences of the NIH under Award Number R43TR001906, OHSU Knight Cancer Institute's Hillcrest Committee Pilot Award, and OHSU Center for Women's Health Circle of Giving Award. Its contents are solely the responsibility of the authors and do not necessarily represent the official views of the NIH. We thank Dr. Sudarshan Anand of OHSU's Cell, 
Developmental and Cancer Biology department for his independent review of the data as required by OHSU's conflict of interest guidelines. Thanks to Dr. John Minna and Dr. Rolf Brekken of UT Southwestern Medical Center for their guidance and helpful advice. We acknowledge expert technical assistance by Drs. Stefanie Kaech Petrie and Felice Kelly of the Advanced Light Microscopy Core in the Department of Neurology and Jungers Center at Oregon Health and Science University. We thank Steven Adamou for electron microscopy performed at the Multiscale Microscopy Core, a member of the OHSU University Shared Resource Cores. We thank Dr. Don Gibbon's lab at MD Anderson Cancer Center for generous gift of LLC-JSP murine lung cancer cell line. Thanks to Drs. Michael Templin and Melinda Tyner at Charles River Laboratory (Ashland, Ohio) and their team for their assistance in designing and executing NHP studies along with data interpretation.

\section{Conflict of Interest.}

OHSU, MR, JWG, and WY have a significant financial interest in PDX Pharmaceuticals, Inc., a company that may have a commercial interest in the results of this research and technology. This potential personal and institutional conflict of interest has been reviewed and managed by OHSU.

\section{Data Availability.}

The data that support the findings of this study are available from the corresponding author upon reasonable request.

\section{References}

1. Ohaegbulam KC, Assal A, Lazar-Molnar E, Yao Y, Zang X. Human cancer immunotherapy with antibodies to the PD-1 and PD-L1 pathway. Trends in molecular medicine 2015; 21(1): 24-33.

2. Shrimali RK, Janik JE, Abu-Eid R, Mkrtichyan M, Khleif SN. Programmed death-1 \& its ligands: promising targets for cancer immunotherapy. Immunotherapy 2015; 7(7): 777-92.

3. Zou W, Wolchok JD, Chen L. PD-L1 (B7-H1) and PD-1 pathway blockade for cancer therapy: Mechanisms, response biomarkers, and combinations. Science Translational Medicine 2016; 8(328): 328rv4-rv4.

4. Reck M, Rodríguez-Abreu D, Robinson AG, et al. Pembrolizumab versus Chemotherapy for PD-L1Positive Non-Small-Cell Lung Cancer. New England Journal of Medicine 2016; 375(19): 1823-33.

5. Malhotra J, Jabbour SK, Aisner J. Current state of immunotherapy for non-small cell lung cancer. Translational lung cancer research 2017; 6(2): 196-211.

6. Moya-Horno I, Viteri S, Karachaliou N, Rosell R. Combination of immunotherapy with targeted therapies in advanced non-small cell lung cancer (NSCLC). Therapeutic advances in medical oncology 2018; 10: 1758834017745012-. 
7. Teng MWL, Ngiow SF, Ribas A, Smyth MJ. Classifying Cancers Based on T-cell Infiltration and PD-L1. Cancer Res 2015; 75(11): 2139-45.

8. Zhuang Y, Liu C, Liu J, Li G. Resistance Mechanism of PD-1/PD-L1 Blockade in the Cancer-Immunity Cycle. Onco Targets Ther 2020; 13: 83-94.

9. Reynolds KL, Cohen JV, Ryan DP, et al. Severe immune-related adverse effects (irAE) requiring hospital admission in patients treated with immune checkpoint inhibitors for advanced malignancy: Temporal trends and clinical significance. Journal of Clinical Oncology 2018; 36(15_suppl): 3096-.

10. Liu Z, Sun Q, Wang X. PLK1, A Potential Target for Cancer Therapy. Translational oncology 2016; 10(1): 22-32.

11. Gutteridge REA, Ndiaye MA, Liu X, Ahmad N. Plk1 Inhibitors in Cancer Therapy: From Laboratory to Clinics. Molecular cancer therapeutics 2016; 15(7): 1427-35.

12. Li M, Liu Z, Wang X. Exploration of the Combination of PLK1 Inhibition with Immunotherapy in Cancer Treatment. Journal of oncology 2018; 2018: 3979527-.

13. Zhang Y, Du XL, Wang CJ, et al. Reciprocal activation between PLK1 and Stat3 contributes to survival and proliferation of esophageal cancer cells. Gastroenterology 2012; 142(3): $521-30 . e 3$.

14. Reda M, Ngamcherdtrakul W, Gu S, et al. PLK1 and EGFR targeted nanoparticle as a radiation sensitizer for non-small cell lung cancer. Cancer letters 2019; 467: 9-18.

15. Yan W, Yu H, Li W, et al. Plk1 promotes the migration of human lung adenocarcinoma epithelial cells via STAT3 signaling. Oncol Lett 2018; 16(5): 6801-7.

16. Ngamcherdtrakul W, Morry J, Gu S, et al. Cationic Polymer Modified Mesoporous Silica Nanoparticles for Targeted siRNA Delivery to HER2 + Breast Cancer. Advanced Functional Materials 2015; 25(18): 2646-59.

17. Morry J, Ngamcherdtrakul W, Gu S, et al. Targeted treatment of metastatic breast cancer by PLK1 siRNA delivered by an antioxidant nanoparticle platform. Molecular Cancer Therapeutics 2017: DOI: 10.1158/535-7163.mct-16-0644.

18. Ngamcherdtrakul W, Bejan DS, Cruz-Muñoz W, et al. Targeted Nanoparticle for Co-delivery of HER2 siRNA and a Taxane to Mirror the Standard Treatment of HER2 + Breast Cancer: Efficacy in Breast Tumor and Brain Metastasis. Small; n/a(n/a): 2107550.

19. McCarroll JA, Dwarte $T$, Baigude $H$, et al. Therapeutic targeting of polo-like kinase 1 using RNAinterfering nanoparticles (iNOPs) for the treatment of non-small cell lung cancer. Oncotarget 2015; 6(14): 12020-34.

20. Ferrarotto R, Goonatilake R, Yoo SY, et al. Epithelial-Mesenchymal Transition Predicts Polo-Like Kinase 1 Inhibitor-Mediated Apoptosis in Non-Small Cell Lung Cancer. Clin Cancer Res 2016; 22(7): 1674-86.

21. Choi M, Kim W, Cheon MG, Lee CW, Kim JE. Polo-like kinase 1 inhibitor BI2536 causes mitotic catastrophe following activation of the spindle assembly checkpoint in non-small cell lung cancer cells. Cancer Lett 2015; 357(2): 591-601. 
22. Wang $Y$, Singh R, Wang L, et al. Polo-like kinase 1 inhibition diminishes acquired resistance to epidermal growth factor receptor inhibition in non-small cell lung cancer with T790M mutations. Oncotarget 2016; 7(30): 47998-8010.

23. Gong W, Song Q, Lu X, et al. Paclitaxel induced B7-H1 expression in cancer cells via the MAPK pathway. Journal of chemotherapy (Florence, Italy) 2011; 23(5): 295-9.

24. Peng J, Hamanishi J, Matsumura N, et al. Chemotherapy Induces Programmed Cell Death-Ligand 1 Overexpression via the Nuclear Factor-kB to Foster an Immunosuppressive Tumor Microenvironment in Ovarian Cancer. Cancer Res 2015; 75(23): 5034-45.

25. Stutvoet TS, Kol A, de Vries EG, et al. MAPK pathway activity plays a key role in PD-L1 expression of lung adenocarcinoma cells. J Pathol 2019; 249(1): 52-64.

26. Zerdes I, Matikas A, Bergh J, Rassidakis GZ, Foukakis T. Genetic, transcriptional and posttranslational regulation of the programmed death protein ligand 1 in cancer: biology and clinical correlations. Oncogene 2018; 37(34): 4639-61.

27. Chen L, Gibbons DL, Goswami S, et al. Metastasis is regulated via microRNA-200/ZEB1 axis control of tumour cell PD-L1 expression and intratumoral immunosuppression. Nature communications 2014; 5: 5241.

28. Gu S, Hu Z, Ngamcherdtrakul W, et al. Therapeutic siRNA for drug-resistant HER2-positive breast cancer. Oncotarget 2016; 7(12): 14727-41.

29. Nel AE, Mädler L, Velegol D, et al. Understanding biophysicochemical interactions at the nano-bio interface. Nature materials 2009; 8(7): 543-57.

30. He Z, Liu K, Manaloto E, et al. Cold Atmospheric Plasma Induces ATP-Dependent Endocytosis of Nanoparticles and Synergistic U373MG Cancer Cell Death. Sci Rep 2018; 8(1): 5298-.

31. Liu X, Lei M, Erikson RL. Normal cells, but not cancer cells, survive severe Plk1 depletion. Mol Cell Biol 2006; 26(6): 2093-108.

32. Rudolph D, Impagnatiello MA, Blaukopf C, et al. Efficacy and mechanism of action of volasertib, a potent and selective inhibitor of Polo-like kinases, in preclinical models of acute myeloid leukemia. $J$ Pharmacol Exp Ther 2015; 352(3): 579-89.

33. Cholewa BD, Ndiaye MA, Huang W, Liu X, Ahmad N. Small molecule inhibition of polo-like kinase 1 by volasertib (BI 6727) causes significant melanoma growth delay and regression in vivo. Cancer Lett 2017; 385: 179-87.

34. Abbou S, Lanvers-Kaminsky C, Daudigeos-Dubus E, et al. Polo-like Kinase Inhibitor Volasertib Exhibits Antitumor Activity and Synergy with Vincristine in Pediatric Malignancies. Anticancer Res 2016; 36(2): 599-609.

35. Du W, Huang H, Sorrelle N, Brekken RA. Sitravatinib potentiates immune checkpoint blockade in refractory cancer models. JCl Insight 2018; 3(21): e124184.

36. Hai J, Zhang H, Zhou J, et al. Generation of Genetically Engineered Mouse Lung Organoid Models for Squamous Cell Lung Cancers Allows for the Study of Combinatorial Immunotherapy. Clinical Cancer Research 2020; 26(13): 3431. 
37. Ehlerding EB, Chen F, Cai W. Biodegradable and Renal Clearable Inorganic Nanoparticles. Advanced science (Weinheim, Baden-Wurttemberg, Germany) 2016; 3(2).

38. Kempen PJ, Greasley S, Parker KA, et al. Theranostic Mesoporous Silica Nanoparticles Biodegrade after Pro-Survival Drug Delivery and Ultrasound/Magnetic Resonance Imaging of Stem Cells. Theranostics 2015; 5(6): 631-42.

39. Ngamcherdtrakul W, Reda M, Nelson MA, et al. In Situ Tumor Vaccination with Nanoparticle CoDelivering CpG and STAT3 siRNA to Effectively Induce Whole-Body Antitumor Immune Response. Advanced Materials 2021; 33(31): 2100628.

40. Yantasee W, Gray JW, Ngamcherdtrakul W. Novel siRNA-nanoparticle platform for treating drug resistant HER2 positive breast cancer. Final Report to the National Cancer Institute $(\mathrm{NCl})$ for the Phase II SBIR Contract \# HHSN261201300078C to PDX Pharmaceuticals in collaboration with the BME of OHSU; September 9, 2016.

41. Gu S, Ngamcherdtrakul W, Reda M, Hu Z, Gray JW, Yantasee W. Lack of acquired resistance in HER2positive breast cancer cells after long-term HER2 siRNA nanoparticle treatment. PloS one 2018; 13(6): e0198141.

42. U.S. Department of Health and Human Services, Food and Drug Administration, Center for Drug Evaluation and Research (CDER). Guidance for Industry Estimating the Maximum Safe Starting Dose in Initial Clinical Trials for Therapeutics in Adult Healthy Volunteers.. Pharmacology \& Toxicology, 2005.

43. Judge AD, Sood V, Shaw JR, Fang D, McClintock K, MacLachlan I. Sequence-dependent stimulation of the mammalian innate immune response by synthetic siRNA. Nature Biotechnology 2005; $23(4)$ : 457-62.

44. Robbins M, Judge A, MacLachlan I. siRNA and innate immunity. Oligonucleotides 2009; 19(2): 89102.

45. Pei $X$, Fan X, Zhang H, et al. Low frequency, weak MCP-1 secretion and exhausted immune status of peripheral monocytes were associated with progression of severe enterovirus A71-infected hand, foot and mouth disease. Clin Exp Immunol 2019; 196(3): 353-63.

46. Ngamcherdtrakul W, Sangvanich T, Reda M, Gu S, Bejan D, Yantasee W. Lyophilization and stability of antibody-conjugated mesoporous silica nanoparticle with cationic polymer and PEG for siRNA delivery. International journal of nanomedicine 2018; 13: 4015-27.

47. American Cancer Society. Cancer Facts \& Figures. 2020.

48. Campbell JD, Alexandrov A, Kim J, et al. Distinct patterns of somatic genome alterations in lung adenocarcinomas and squamous cell carcinomas. Nature genetics 2016; 48(6): 607-16.

49. Rizvi NA, Hellmann MD, Snyder A, et al. Mutational landscape determines sensitivity to PD-1 blockade in non-small cell lung cancer. Science 2015; 348(6230): 124-8.

50. Zhang J, Bu X, Wang H, et al. Cyclin D-CDK4 kinase destabilizes PD-L1 via cullin 3-SPOP to control cancer immune surveillance. Nature 2018; 553(7686): 91-5. 
51. Jiao S, Xia W, Yamaguchi H, et al. PARP Inhibitor Upregulates PD-L1 Expression and Enhances Cancer-Associated Immunosuppression. Clin Cancer Res 2017; 23(14): 3711-20.

52. Esteva FJ, Hubbard-Lucey VM, Tang J, Pusztai L. Immunotherapy and targeted therapy combinations in metastatic breast cancer. The Lancet Oncology 2019; 20(3): e175-e86.

53. Matheu MP, Sen D, Cahalan MD, Parker I. Generation of bone marrow derived murine dendritic cells for use in 2-photon imaging. J Vis Exp 2008; (17): 773.

54. Kimura A, Naka T, Kishimoto T. IL-6-dependent and -independent pathways in the development of interleukin 17-producing T helper cells. Proceedings of the National Academy of Sciences 2007; 104(29): 12099.

\section{Figures}

A

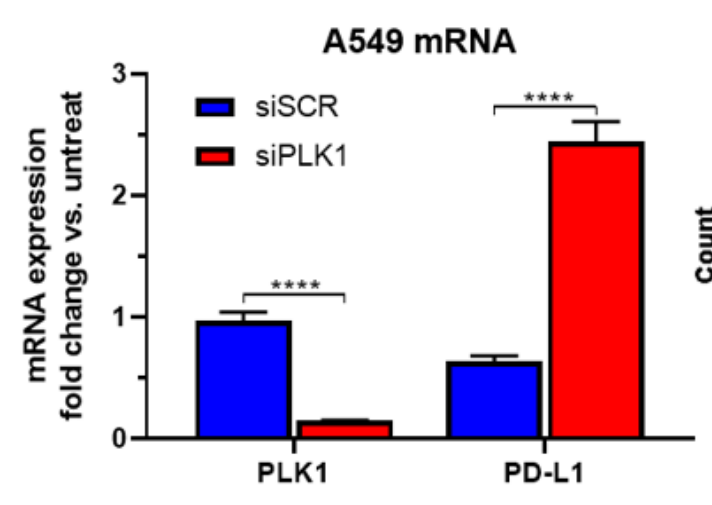

B

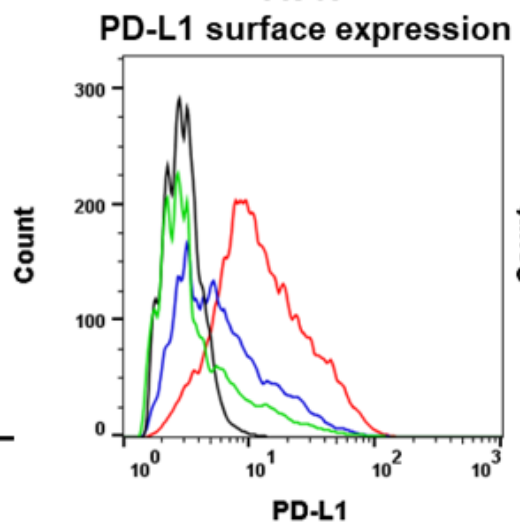

C

LLC-JSP

PD-L1 surface expression

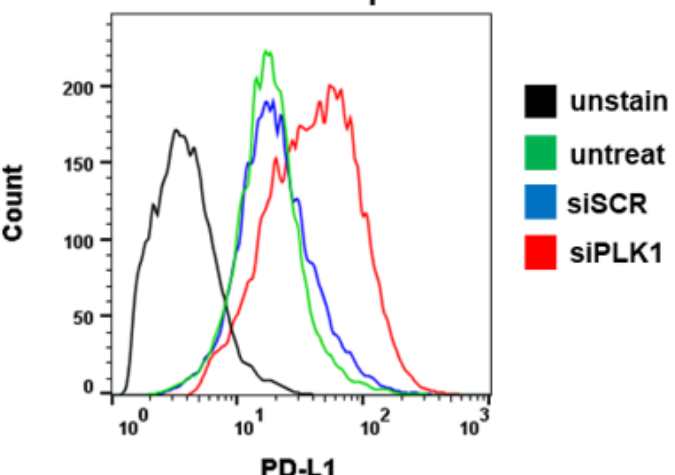

\section{Figure 1}

PLK1 knockdown by siRNA induces PD-L1 expression. (A) PLK1 and PD-L1 mRNA expression in A549 (human NSCLC) at $48 \mathrm{hr}$ post treatment with PLK1 siRNA (siPLK1) or scrambled siRNA (siSCR) normalized to HPRT housekeeping gene. Dharmafect 1 transfection agent was used. Data presented as mean \pm SD from triplicates; $* \star \star \star P<0.0001$. PD-L1 surface expression of (B) A549 and (C) LLC-JSP at $72 \mathrm{hr}$ post treatments assessed by flow cytometry (10,000 events per sample). 
A

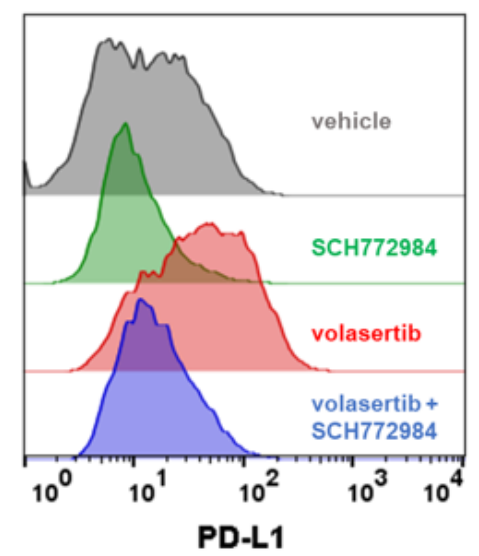

PD-L1 expression

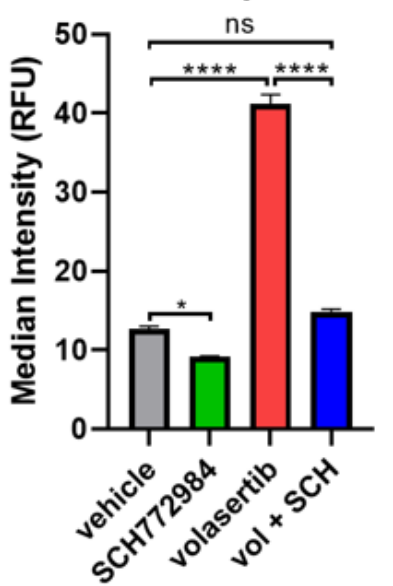

B

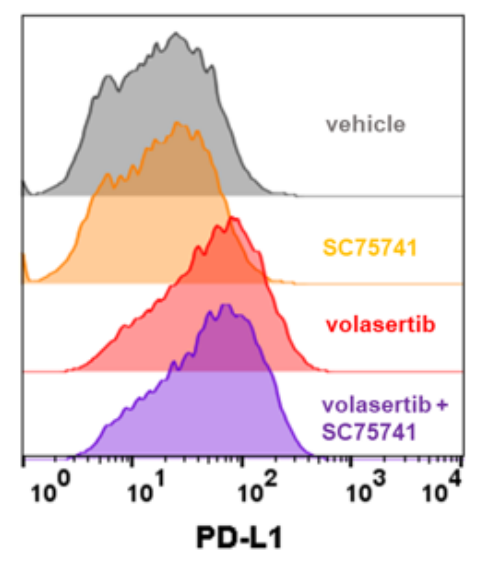

PD-L1 expression

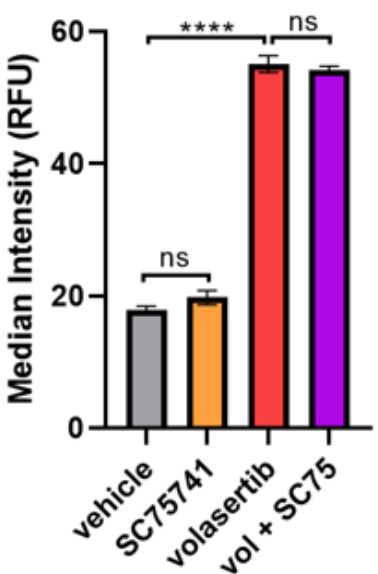

Figure 2

Volasertib-induced PD-L1 upregulation is dependent on MAPK. (A) PD-L1 surface expression of NSCLC cells (H460) treated with vehicle control (0.1\% DMSO in PBS), SCH772984 (ERK1/2 small molecule inhibitor, 1 uM), volasertib (100 nM), or SCH772984 (SCH; 1 uM) + volasertib (vol; 100 nM) - (left) representative histograms, (right) MFI quantification. (B) PD-L1 surface expression of $\mathrm{H} 460$ cells treated with vehicle control (0.1\% DMSO in PBS), SC75741 (NF-kB inhibitor, $1 \mathrm{uM}$ ), volasertib (100 nM), or SC75741 (SC75; 1 uM) + volasertib (vol; 100 nM) - (left) representative histograms, (right) MFI quantification. Data presented as mean \pm SD from biological duplicates, 10,000 events per sample; ${ }^{*} \mathrm{P}<0.05,{ }^{* \star * *} \mathrm{P}<0.0001, \mathrm{~ns}-$ not significant.

A

LLC-JSP flank $200 \mathrm{~K}$ cells
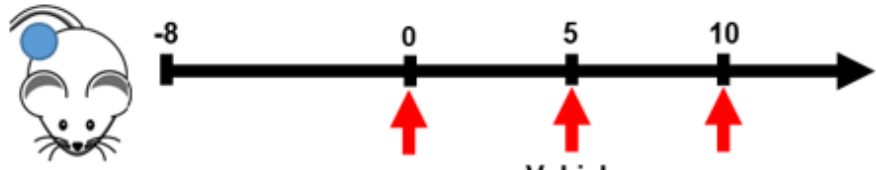

Vehicle

Volasertib

PD-L1 antibody

Volasertib + PD-L1 antibody
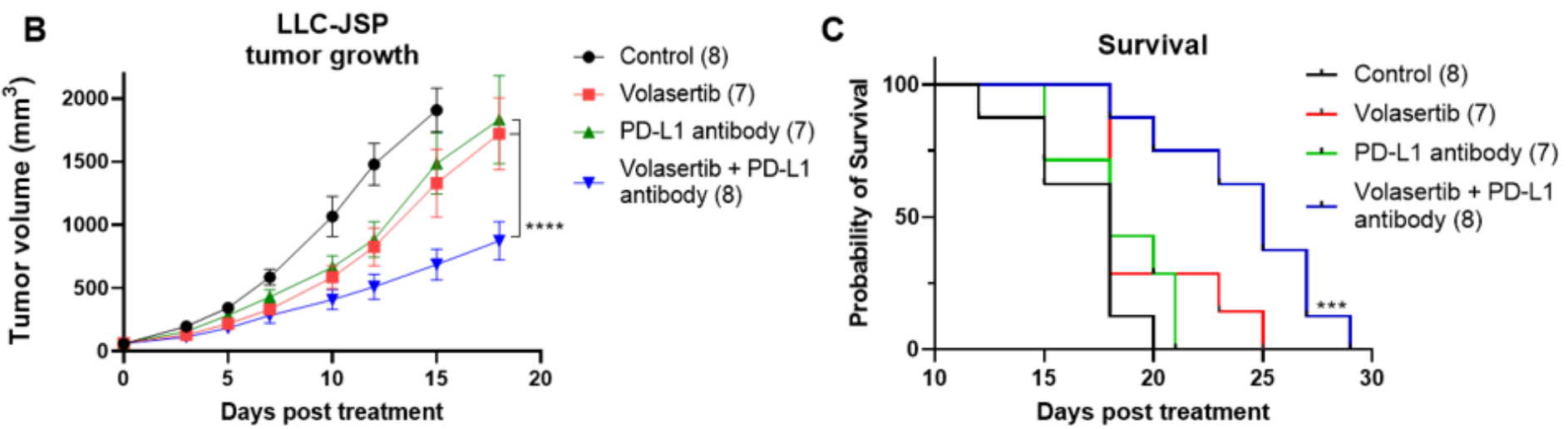
Figure 3

PLK1 inhibition potentiates PD-L1 blockade in syngeneic lung tumors. (A) C57BL/ 6 mice were injected with 200,000 LLC-JSP cells in right flank. On day 0 (8 post tumor inoculation), mice were grouped ( $n=7-8)$ and received i.p. treatments of control vehicles (PBS and $\mathrm{HCl} /$ saline), PLK1 inhibitor volasertib (20 $\mathrm{mg} / \mathrm{kg}$ ), PD-L1 antibody (10 mg/kg), or combination of PLK1 inhibitor and PD-L1 antibody at the same dose. Treatments were administered every 5 days for 3 doses. (B) Tumor growth of mice. Data presented as mean \pm SEM; ${ }^{* \star * P} P<0.001,{ }^{* \star \star * P} P<0.0001$. (C) Kaplan-Meier Survival curve. ${ }^{* \star * P} P<0.001$ vs. saline.
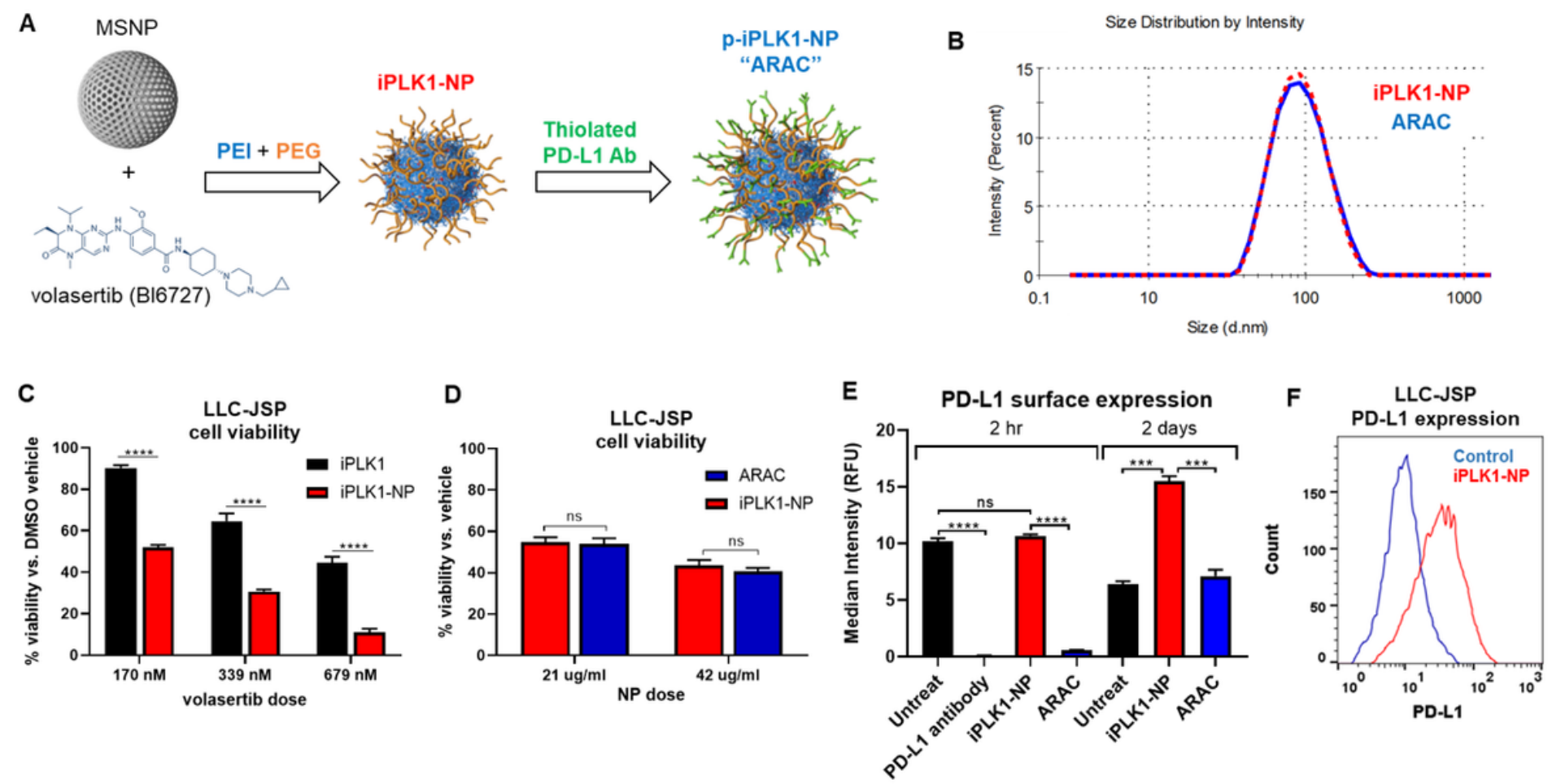

Figure 4

PD-L1 antibody conjugated NP for delivering PLK1 inhibitor volasertib (p-iPLK1-NP; ARAC). (A) Synthesis scheme. (B) Hydrodynamic size by dynamic light scattering (DLS). (C) Cell viability by CTG of LLC-JSP cells treated with volasertib (iPLK1) or iPLK1-NP; all having equivalent volasertib dose as specified. (D) Cell viability by CTG of LLC-JSP cells treated with iPLK1-NP or ARAC (p-iPLK1-NP) at equivalent NP and volasertib dose. Data presented as mean \pm SD from 4 independent samples; $\star \star \star \star P<0.0001$, ns - not significant. (E\&F) PD-L1 surface expression of the cells treated with PBS, PD-L1 antibody $(50 \mu \mathrm{g} / \mathrm{ml})$, iPLK1-NP (with $210 \mathrm{ng} / \mathrm{ml}$ volasertib), or ARAC (with $210 \mathrm{ng} / \mathrm{ml}$ volasertib and $1.68 \mu \mathrm{g} / \mathrm{ml}$ antibody) for 2 hrs and 2 days. Data presented as mean \pm SD from biological duplicates, 10,000 events per sample; $\star \star \star * P<0.0001$, ns - not significant. 


\section{A}
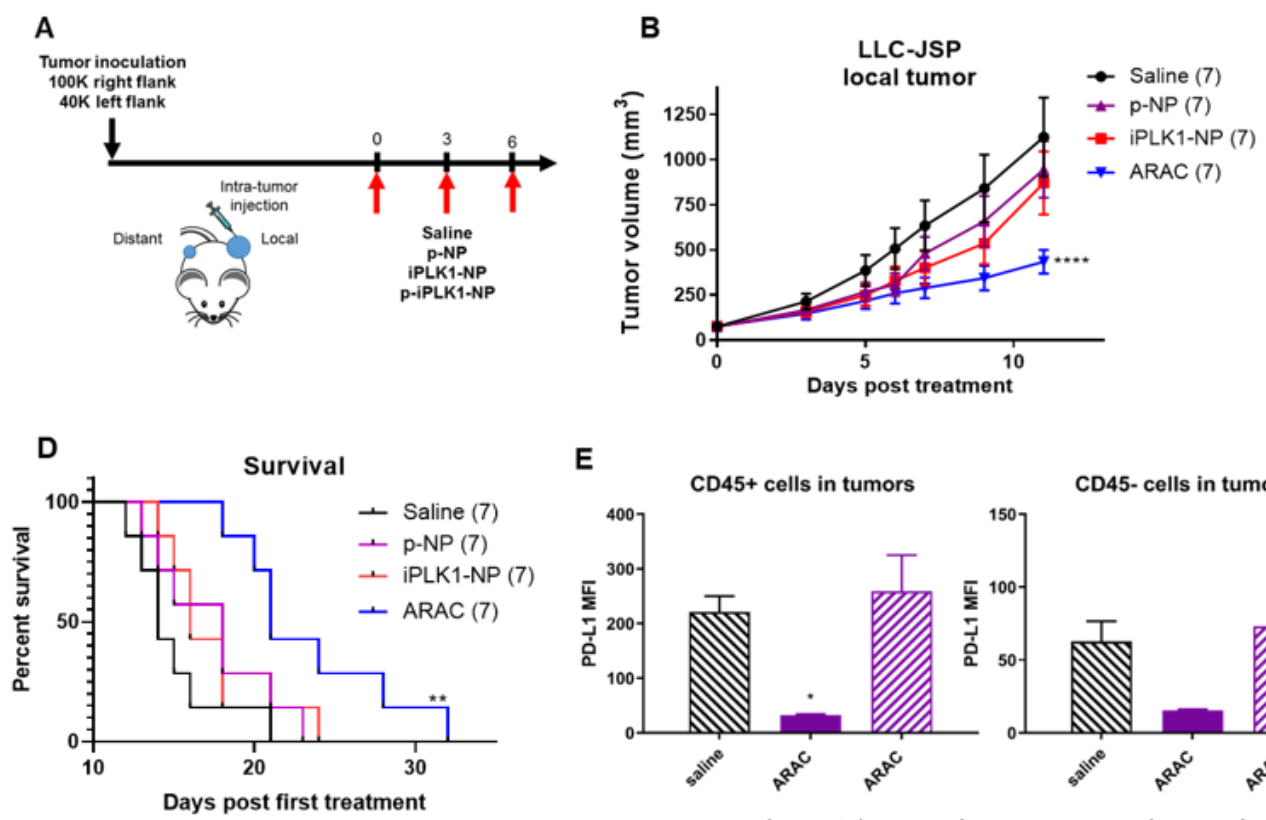

G

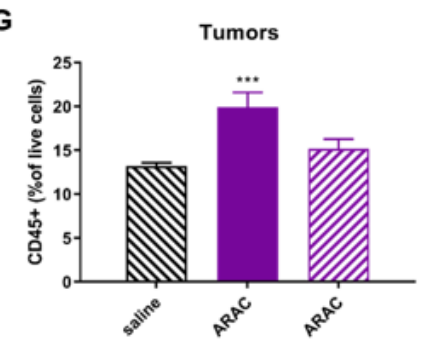

$\vdash$ local + distant $\dashv$
E

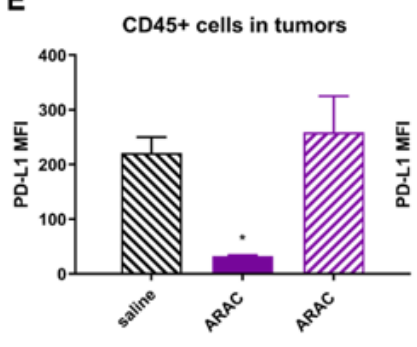

$\vdash$ local + distant -1

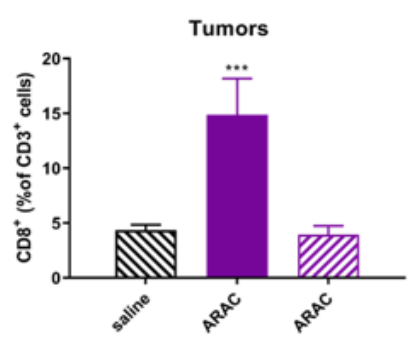

$\vdash$ local + distant $\dashv$

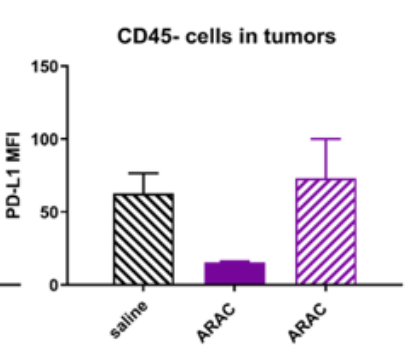

$\vdash$ local + distant $\dashv$

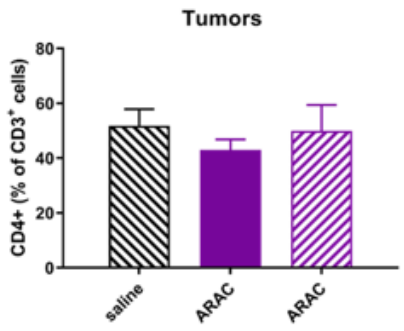

$\vdash$ local + distant $\dashv$
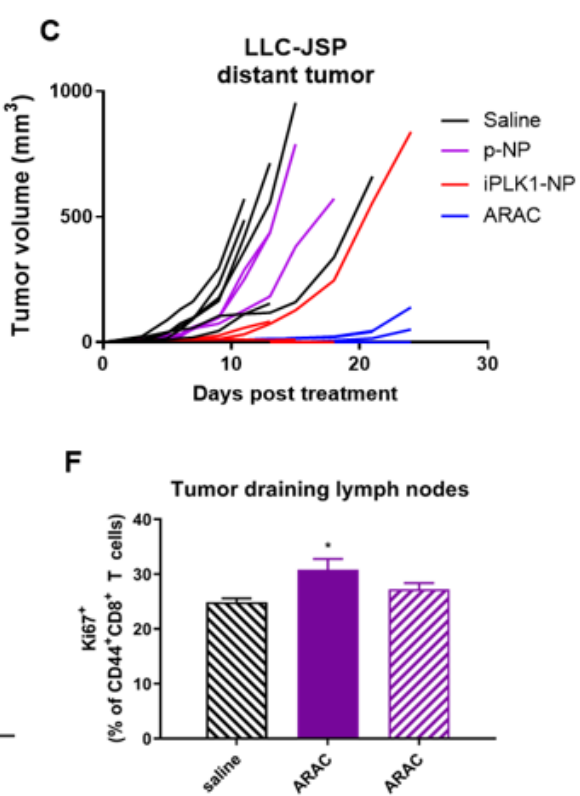

$\vdash$ local + distant -1

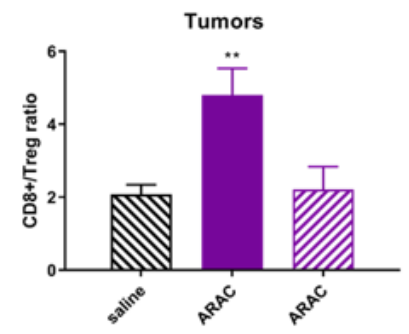

- local + distant -1

Figure 5

ARAC elicits anti-tumor immune effects. (A) 100,000 LLC-JSP cells were injected in right flank and 40,000 cells were injected in left flank of C57BL/ 6 mice. On day 12 post tumor inoculation, mice $(n=7$ per treatment group) received intratumoral treatments of saline, p-NP, iPLK1-NP, or ARAC to the right (local) tumor. $0.5 \mathrm{mg} \mathrm{NP}$ (containing $2.5 \mu \mathrm{g}$ volasertib and/or $20 \mu \mathrm{g}$ PD-L1 antibody) in $50 \mu \mathrm{l}$ per dose for 3 doses total. (B) Local (treated) tumor growth. (C) Distant (untreated) tumor growth of individual mice (distant tumors developed in 6/7 saline, 3/7 p-NP, 3/7 iPLK1-NP, and 2/7 ARAC at shown time-points). (D) Kaplan Meier Survival curve (mice were euthanized when a combined tumor size reached $\left.2000 \mathrm{~mm}^{3}\right)$. (EG) Mice $(n=7)$ were inoculated with 250,000 and 100,000 LLC-JSP cells for bilateral tumors and treated as shown in (A) with ARAC or saline. One day post $3^{\text {rd }}$ injection, tumors were harvested and processed into single cell suspensions for flow cytometry analysis. (E) PD-L1 expression (median fluorescent intensity; MFI) in CD45+ and CD45- cells. (F) Proliferative effector T cells (\%Ki67 of CD44+CD8+ cells) in tumor draining lymph nodes. (G) CD45+ (\% of live cells), CD8+ (\% of CD45+CD3+ cells), CD 4+ (\% of CD45+CD3+ cells), and CD8+/Treg (Regulatory T cells; CD4+FoxP3+ (\% of CD45+CD3+ cells) in tumors. Data presented as mean $\pm S E M ; * P<0.05, \star \star P<0.01,{ }^{\star} * \star P<0.001, \star \star \star \star * P<0.0001$. 
A

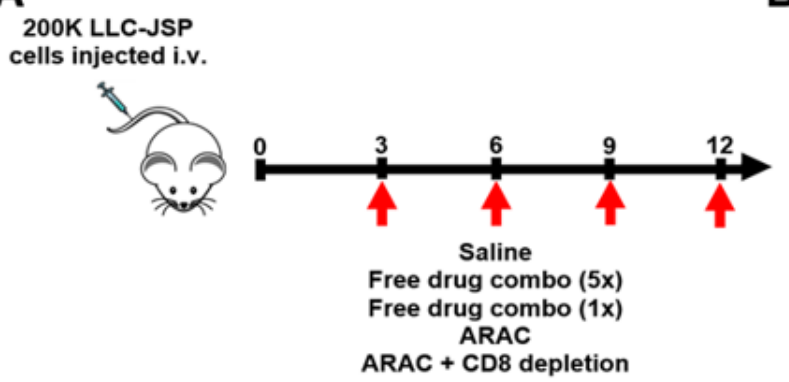

C

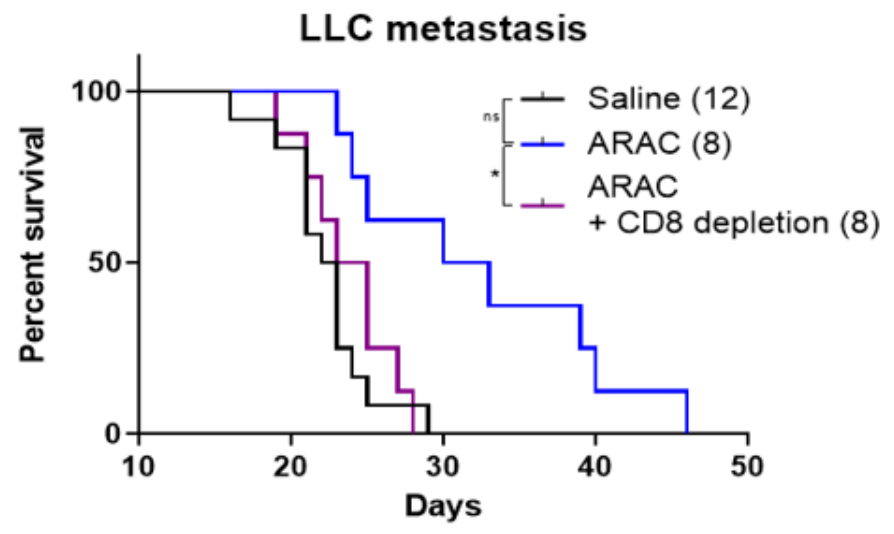

B

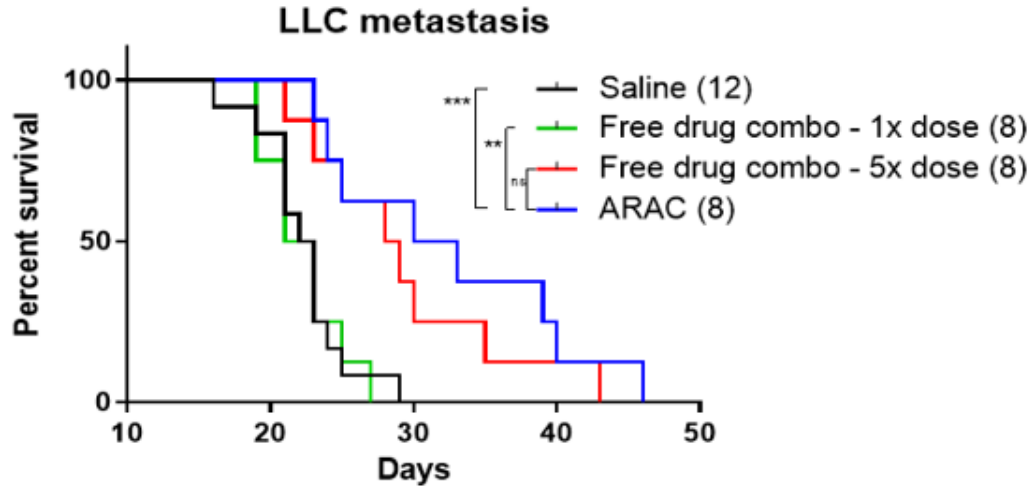

D

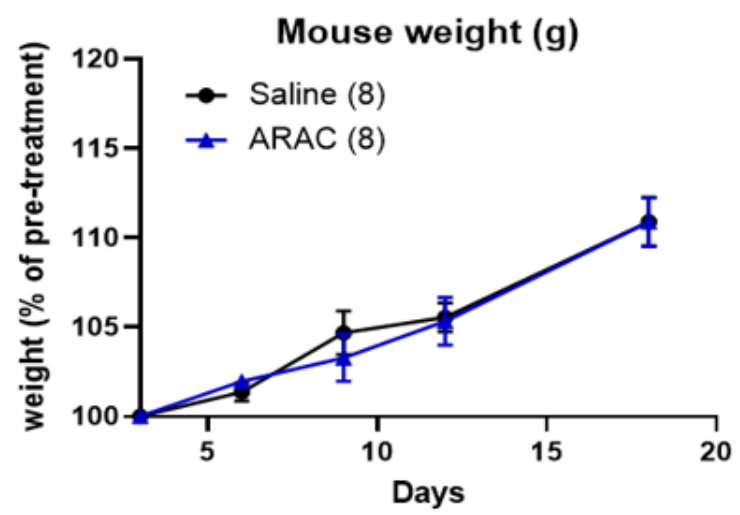

Figure 6

ARAC improves survival of mice bearing metastatic lung tumors. (A) C57BL/ 6 mice were injected with 200,000 LLC-JSP cells intravenously. After 3 days, mice were treated with saline, free drugs (i.p., at $1 \mathrm{x}$ dose: $2.5 \mu \mathrm{g}$ volasertib and $20 \mu \mathrm{g}$ PD-L1 and 5x dose: $12.5 \mu \mathrm{g}$ volasertib and $100 \mu \mathrm{g}$ PD-L1 antibody), ARAC (i.v., containing $2.5 \mu \mathrm{g}$ volasertib and $20 \mu \mathrm{g}$ PD-L1), or ARAC + anti-CD8 (200 mg i.p. twice weekly). $(B, C)$ Kaplan-Meier Survival curve. ${ }^{*} P<0.05,{ }^{* \star} P<0.01$, ${ }^{\star \star \star} P<0.001$ (Log-rank Mantel-Cox test). (D) Mouse weight change post first treatment; data presented as mean \pm SEM.

A

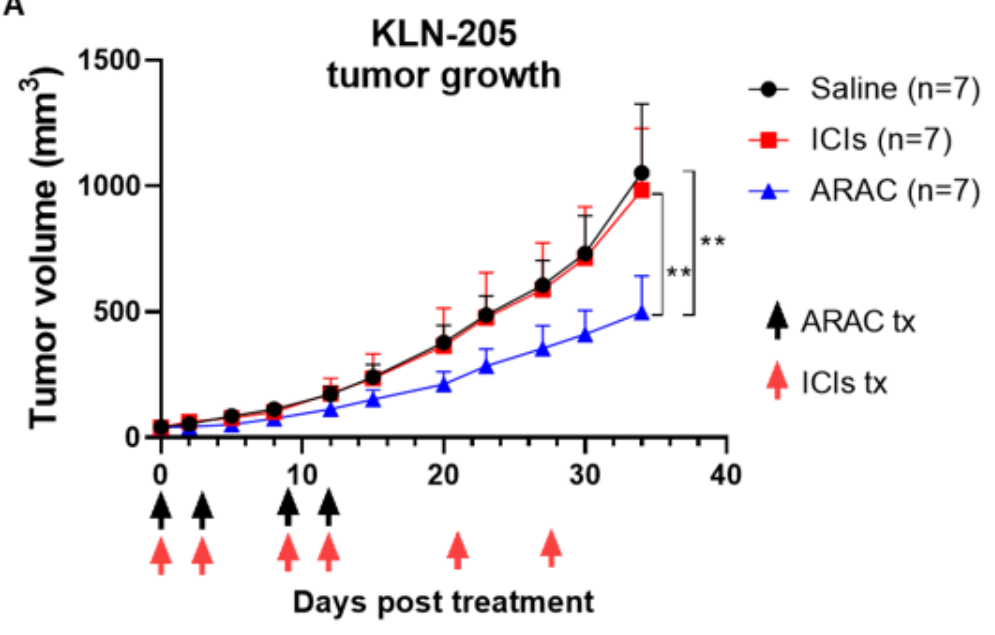

B

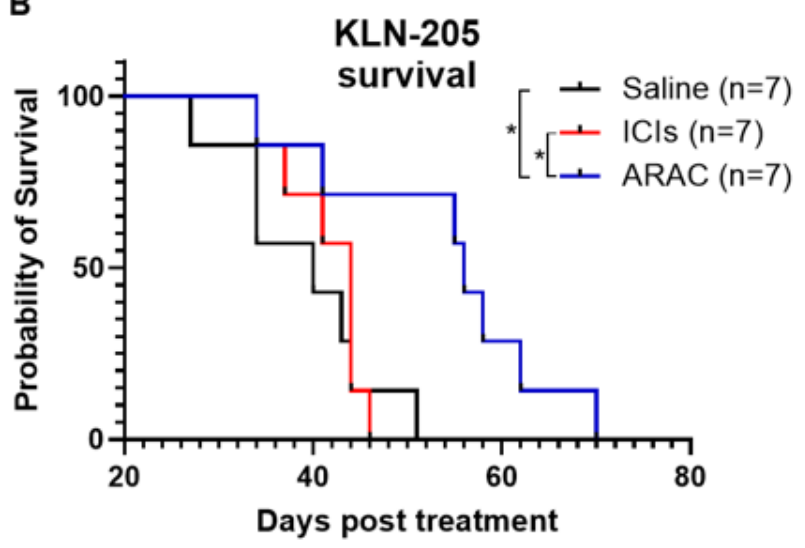




\section{Figure 7}

ARAC reduces tumor growth and prolongs survival of mice bearing $\mathrm{KLN}-205$ murine lung tumors. (A) DBA/2 mice were injected with 500,000 KLN-205 cells in 100 uL PBS on the right flank. 13 days post inoculation, mice were grouped $(n=7)$ and treated with saline, ICls (PD-1 and CTLA-4 antibodies - i.p. 200 $\mathrm{mg} /$ dose and $100 \mathrm{mg} /$ dose respectively - for 6 doses), or ARAC (i.v. $50 \mathrm{mg} / \mathrm{kg}$ for 4 doses). (A) KLN-205 tumor growth; black arrows specify ARAC dosing days, red arrows specify ICl dosing days. Data presented as mean $+S E M$; ${ }^{*} P<0.01$ (B) Kaplan-Meier Survival curve. ${ }^{*} P<0.05$, (Log-rank Mantel-Cox test).

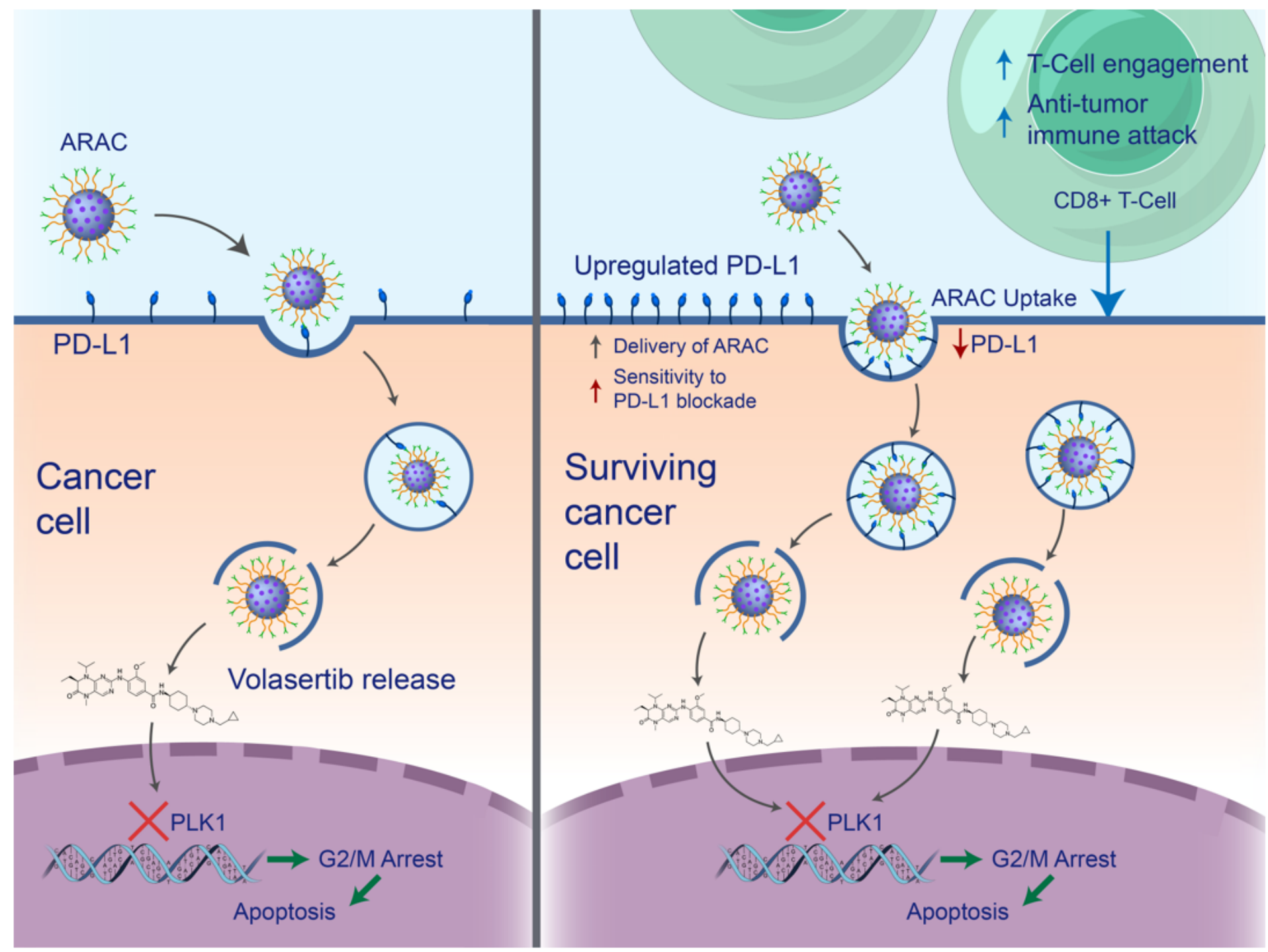

\section{Figure 8}

Scheme 1: Proposed mechanism of action of ARAC nanoconstruct. (Left cell) ARAC binds to PD-L1 on cancer cell surface and is internalized via receptor-mediated endocytosis. Endosomal escape of ARAC is mediated by PEI polymer ${ }^{16}$ and volasertib is released to inhibit PLK1 activity, leading to G2/M cell cycle arrest and apoptotic cell death. However, G2/M arrest induced by volasertib upregulates PD-L1 levels in surviving cancer cells thereby rendering them unresponsive to immune-mediated effects (due to PD-L1- 
mediated immunosuppression). (Right cell) We capitalize on this property by utilizing elevated PD-L1 levels in surviving cancer cells as the homing target for subsequent ARAC delivery, leading to cancer targeting in a feed-forward manner (i.e., higher targeting with increased doses of the treatment).

Enhanced delivery of ARAC results in loss of PD-L1, which allows for cytotoxic CD8+ T cells to effectively kill cancer and generate an anti-tumor immune response.

\section{Supplementary Files}

This is a list of supplementary files associated with this preprint. Click to download.

- SupplementaryTablesrevision.xlsx

- SupplementaryFiguresrevision.docx 